\title{
LOCALIZATION FOR LOGARITHMIC STABLE MAPS
}

\author{
S. MOLCHO AND E. ROUTIS
}

\begin{abstract}
We prove a virtual localization formula for Bumsig Kim's space of Logarithmic Stable Maps. The formula is closely related and can in fact recover the relative virtual localization formula of Graber-Vakil.
\end{abstract}

\section{INTRODUCTION AND BACKGROUND}

In his papers Li01 and Li02, Jun Li introduced and studied the space of relative stable maps. We recall the setup: fix a pair $(X, D)$ of a smooth variety with a smooth divisor and discrete data $\Gamma=\left(g, \vec{c}=\left(c_{1}, \cdots, c_{h}\right), \beta\right)$, consisting of the arithmetic genus $g$ of a nodal curve, a vector $\vec{c}$ of integers, and a homology class $\beta$ in $X$. We wish to parametrize stable maps $f:(C, \vec{y}, \vec{x}) \rightarrow X$ from a genus $g$ nodal curve $C$ with two sets of marked points $\vec{y}=\left(y_{1}, \cdots, y_{m}\right)$ and $\vec{x}=\left(x_{1}, \cdots, x_{h}\right)$ into the variety $X$, whose image lies in the given homology class and with prescribed incidence conditions along the divisor, namely, $f^{-1}(D)=\sum c_{i} x_{i}$.

The moduli space parametrizing is not proper: a limit of such maps may fail to exist, as in the limit that the whole curve may lie entirely in the divisor $D$. Jun Li and, at about the same time, Li-Ruan [LR01] and Ionel-Parker [P03, IP04] from the point of view of symplectic Gromov-Witten theory, gave a beautiful solution to this issue. Jun Li's idea can be outlined as follows. When a limit of maps tends to collapse into the divisor, the space $X$ sprouts a new component, which is isomorphic to the projective completion of the normal bundle $\mathbb{P}\left(N_{D / X} \oplus 1\right)$ of $D$ to contain the image, in a manner similar to a blowup. We then require that the prescribed behavior along the divisor does not happen along the original divisor $D$, but rather the divisor at infinity in $\mathbb{P}\left(N_{D / X} \oplus 1\right)$, which we denote $D[1]$. We call $X$ with this new component $X[1]$; we then have a new pair $(X[1], D[1])$ and we may consider stable maps as above to this pair. When a family of maps to $X[1]$ tends to collapse into $D[1]$, the variety $X[1]$ sprouts a new component that replaces $D[1]$, as above, to create a new space $X[2]$ with a divisor $D[2]$ at infinity, and so forth. In general, a pair $(X[n], D[n])$ is constructed from the pair $(X[n-$ $1], D[n-1])$ inductively. It is called the $n$-th expansion of $(X, D)$. Li's moduli space $\mathcal{M}_{\Gamma}(X, D)$ parametrizes stable maps whose target is allowed to be any of the

\footnotetext{
Received by the editors November 19, 2015, and, in revised form, April 10, 2018 and July 16, 2018 .

2010 Mathematics Subject Classification. Primary 14N35; Secondary 14D23.

The second author was supported by the World Premier International Research Center Initiative (WPI), MEXT, Japan.
}

(C)2019 by the authors under Creative Commons Attribution-Noncommercial 3.0 License (CC BY NC 3.0) 
expansions $(X[n], D[n])$ above, with prescribed behavior along the divisor at infinity $D[n]$ and with a certain compatibility requirement along the divisor $D[k], k<n$ : only nodes of the source curve can map to $D[k]$, and when a node maps to $D[k]$, the two components of the curve containing the node have the same contact order with the divisor $D[k]$; this is called the predeformability or "kissing" condition. This space is proper and is shown to carry a virtual fundamental class, so one can define in a standard manner a type of Gromov-Witten invariant for $(X, D)$, called relative Gromov-Witten invariants. For details of the construction, the reader should consult Jun Li's original paper [Li01].

Jun Li also considers a variant of this situation, where instead of a pair $(X, D)$ we consider a semistable nodal variety of the form $X=Y_{1} \cup_{D} Y_{2}$. This means that $X$ is the union of two smooth varieties $Y_{i}$ along a common smooth divisor $D$ in both of them that satisfies the following technical condition on the normal bundles: $N_{D / Y_{1}} \otimes N_{D / Y_{2}} \cong 1$. Stable maps into $X$ must satisfy a similar predeformability condition as above, and the space is compactified by allowing the targets to vary as before. $X$ may deform to a target $X[1]$, where $D$ is replaced by $\mathbb{P}\left(N_{D / Y_{1}} \oplus 1\right) \cong$ $\mathbb{P}\left(N_{D / Y_{2}} \oplus 1\right)$, with $Y_{1}$ glued along the 0 section and $Y_{2}$ along the infinity section, $X[1]$ may deform to $X[2]$ where the divisor connection $Y_{2}$ with $\mathbb{P}\left(N_{D / X} \oplus 1\right)$ is replaced by another copy of $\mathbb{P}\left(N_{D / X} \oplus 1\right)$, and so forth. The spaces $X[n]$ are called the expanded degenerations of $X$. The space of expanded degenerations also carries a virtual fundamental class, and one is thus able to extend the notion of GromovWitten invariants for targets $X=Y_{1} \cup Y_{2}$, which are mildly singular. These are the correct Gromov-Witten invariants, in the sense that they satisfy deformation invarance: If $W \rightarrow B$ is a family with smooth total space, smooth general fiber, and central fiber $X=Y_{1} \cup_{D} Y_{2}$, the Gromov-Witten invariants of $X$ as defined by Jun Li coincide with the usual Gromov-Witten invariants of the general fiber, at least when such a comparison makes sense, i.e., for homology classes restricted from $W$.

The relative Gromov-Witten invariants are related to the singular GromovWitten invariants by the degeneration formula. This was also proven by Jun Li and had also been previously considered in the symplectic category in the work of Li-Ruan [LR01] and Ionel-Parker [IP04]. The degeneration formula allows one to compute Gromov-Witten invariants of expanded degenerations from the relative ones and the combinatorics of the expansions. This can be useful because it is often possible to degenerate a smooth variety into a semistable one with very simple components $Y_{i}$. Thus one can calculate Gromov-Witten invariants from relative Gromov-Witten invariants of simpler targets.

Computations of relative Gromov-Witten invariants can still be difficult, as calculations in Gromov-Witten theory often are, even if the targets are very simple. These calculations can be greatly facilitated by the use of Atiyah-Bott localization. Localization formulas for the spaces $\mathcal{M}_{\Gamma}(X, D)$ were established by Graber-Vakil in GV05. The applications of such formulas are far reaching: for example, in GV05, as applications of the formulas the authors recover the ELSV formula and certain striking results about the tautological ring.

Jun Li's constructions are beautiful and geometrically transparent, but suffer from one technical drawback. The virtual fundamental classes defined are hard to work with. The reason for this is that the space of relative stable maps is not an open subset of all maps, but rather, it is locally closed. The perfect obstruction 
theory used to define the virtual fundamental class is thus constructed by hand and not by standard machinery. This is the main reason the paper GV05] is technically difficult.

One way to avoid this issue is to use a different compactification of the space of maps to the pair $(X, D)$ or $Y_{1} \cup_{D} Y_{2}$, by endowing the sources and targets of all maps with logaritmic structures and requiring that the maps between them are log maps. We will explain this more precisely in what follows, but here we would like to remark that this idea agrees with a general philosophy in the modern theory of moduli that states that instead of compactifying a moduli space of certain objects, one may try to build the moduli space of such objects with logarithmic structures. Since logarithmic structures allow mild singularities, this moduli space is often already proper. The space of logarithmic stable maps was constructed by B. Kim in his paper [Kim10]. Kim's space is shown to be an open substack of the space of all logarithmic maps and thus carries a natural virtual fundamental class by restriction, which is simpler than the virtual fundamental class of $\mathcal{M}_{\Gamma}(X, D)$ : its formal properties are almost identical to the fundamental class in the classical Gromov-Witten theory of smooth targets. The situation may be summarized pictorially as follows:

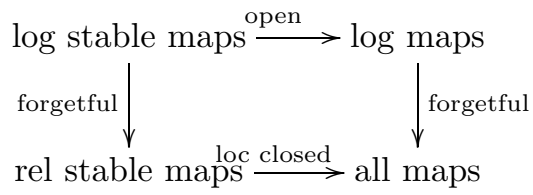

In this paper, we derive an analogous localization formula for the space $\mathcal{M}_{\Gamma}^{\log }(\mathcal{U} / \mathcal{B})$ of logarithmic stable maps. The formula is analogous to the formula of [GV05, but its derivation is closer in spirit with the proofs of localization in classical Gromov-Witten theory, as in [Kon95, GP99. Specifically, we show the following.

Theorem 3.1. $\mathcal{M}_{\Gamma}^{\log }(\mathcal{U} / \mathcal{B})$ is a global quotient stack and admits a $\mathbb{C}^{*}$ equivariant immersion to a smooth Deligne-Mumford stack.

This in particular shows that $\mathcal{M}_{\Gamma}^{\log }(\mathcal{U} / \mathcal{B})$ admits a localization formula. Then, following the work of Graber-Vakil GV05, we obtain explicitly that for suitable splittings of the discrete data $\Gamma$ into subsets $\Gamma_{1}, \Gamma_{2}$, we have the following.

Theorem 5.1 (Log Virtual Localization).

$$
\begin{aligned}
{\left[\mathcal{M}_{\Gamma}^{\log }(\mathcal{U} / \mathcal{B})\right]^{\text {vir }}=} & {\left[\mathcal{M}_{\Gamma}^{\log }(\mathcal{U} / \mathcal{B})^{\operatorname{sim}}\right]^{\text {vir }} } \\
& +\sum_{\Gamma_{1}, \Gamma_{2}} \frac{q_{12 *} p_{12}^{*} \Delta^{!}\left(\left[\mathcal{M}_{\Gamma_{1}}^{\log }(\mathcal{U} / \mathcal{B})^{\operatorname{sim}}\right]^{\operatorname{vir}} \times\left[\mathcal{M}_{\Gamma_{2}}^{\log }(\mathcal{U} / \mathcal{B})^{\sim}\right]^{\text {vir }}\right)}{\left|\operatorname{Aut}\left(\Gamma_{1}, \Gamma_{2}\right)\right|\left(\frac{w-\psi}{d}\right) e^{T}\left(N_{\Gamma_{1}}\right)}
\end{aligned}
$$

The formula is essentially the same as the relative virtual localization formula of GV05]. The difference is that the stacks of simple relative maps and unrigidified relative stable maps of GV05 are replaced by their logarithmic analogues. These are defined carefully in section 5 . In fact, the log virtual localization formula can be used to recover the formula of [GV05]; this is our Corollary [5.4. 


\section{LOGARITHMIC STABLE MAPS}

For completeness, we will recall here the necessary definitions and constructions that we will use. For proofs and more detailed explanations the reader should consult Kim's paper Kim10.

A family of $n$-marked prestable curves, $C \rightarrow S$, carries a canonical structure of a logarithmic map, as shown in F. Kato's paper [Kat00. (see also Ols07]). The log structures and morphisms are defined as follows. The curve $C \rightarrow S$ corresponds to a diagram

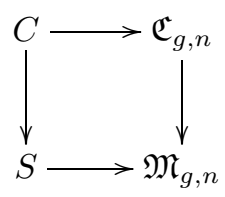

where $\mathfrak{M}_{g, n}$ and $\mathfrak{C}_{g, n}$ is the moduli stack of $n$-marked prestable curves and its universal family respectively. Both stacks carry natural logarithmic structures: in $\mathfrak{M}_{g, n}$ the log structure is given by the divisor corresponding to singular curves, and in $\mathfrak{C}_{g, n}$ the log structure is given by the divisors corresponding to singular curves and the markings. The $\log$ structures on $C$ and $S$ are the ones pulled back from $\mathfrak{C}_{g, n}$ and $\mathfrak{M}_{g, n}$ respectively; we denote the log structure on $S$ by $N^{C / S}$ and on $C$ by $M^{C / S}$ and refer to them as the canonical log structures. The morphism $C \rightarrow S$ is automatically a log morphism. An explicit description of the log structures when $S=$ Spec $k$ is a geometric point can be given in terms of charts as follows: $N^{C / S}$ has a chart isomorphic to $\mathbb{N}^{m}, m=$ the number of nodes of $C ; M^{C / S}=N^{C / S}$ at smooth points; $M^{C / S}=N^{C / S} \oplus \mathbb{N}$ at a marked point; and at a node $M^{C / S}$ is given by the following pushout diagram:

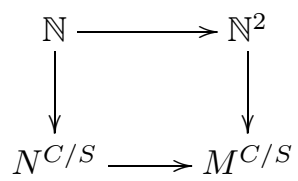

where the horizontal map is the diagonal and the vertical map is the inclusion corresponding to the appropriate node.

Definition 2.1. A genus $g, n$-marked log curve is a morphism $f:(C, M) \rightarrow(S, N)$ of $\log$ schemes such that $C \rightarrow S$ is a family of genus $g, n$-marked prestable curves and the morphism $f$ is obtained from a cartesian diagram of the form

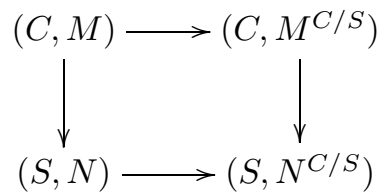

where the horizontal maps are the identities on underlying schemes. Therefore, a $\log$ curve is the same thing as the choice of a prestable curve $C \rightarrow S$ and the choice of a homomorphism of $\log$ structures $s^{C / S}: N^{C / S} \rightarrow N$. We will reserve the notation $s^{C / S}$ to always refer to this homomorphism and denote it by $s$ to simplify notation if no confusion may arise.

We denote the stack parametrizing log curves by $\mathfrak{M}_{g, n}^{\log }$. 
Definition 2.2. A log curve $(M, C) \rightarrow(S, N)$ is called minimal if the log structure $N$ is locally free and there is no locally free submonoid $N^{\prime} \subset N$ that contains the image of $N^{C / S}$.

Here, we call a log structure locally free if around every point it has a chart isomorphic to $\mathbb{N}^{r}$ for some $r$, possibly depending on the point. For example, over $S=$ Spec $k$, where $N^{C / S}=\mathbb{N}^{m}$, all surjections $\mathbb{N}^{m} \rightarrow \mathbb{N}^{r}, r \leq m$, give minimal log curves but no map $\mathbb{N}^{m} \rightarrow \mathbb{N}^{r}, r>m$.

Minimal log curves are essentially the sources of log stable maps; for a more precise statement, see Definition 2.5. Next, we discuss the possible targets, which Kim calls "extended log twisted Fulton-Macpherson type spaces".

Fix a smooth projective variety $X$.

Definition 2.3. A family of schemes or algebraic spaces $W \rightarrow S$ is called a log FM type space of $X$ if, at every point $s \in S$, there is étale locally an étale map

$$
W_{\bar{s}} \rightarrow \operatorname{Spec} k(\bar{s})\left[x, y, z_{1}, \cdots, z_{r-1}\right] /(x y) .
$$

These families of spaces are required to admit canonical log structures $N^{W / S}$ on $S$ and $M^{W / S}$ on $W$ such that $M^{W / S}$ is given by the cocartesian diagram

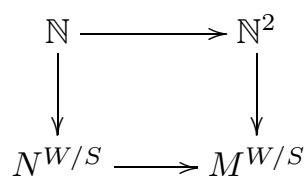

and such that the morphism $W \rightarrow S$ is in fact a $\log$ morphism $\left(W, M^{W / S}\right) \rightarrow$ $\left(S, N^{W / S}\right)$. We will further require that $N^{W / S}$ be locally free. Its rank at $s \in S$ equals the number of irreducible components of the singular locus of the fiber $W_{s}$. We further require that the spaces $W$ come equipped with a map $W \rightarrow X$.

Definition 2.4. An extended log twisted FM type space of $X$ is a log morphism $(W, M) \rightarrow(S, N)$, where

- $W \rightarrow S$ is as in Definition 2.3 above, and all relevant logarithmic data are obtained from a cartesian diagram

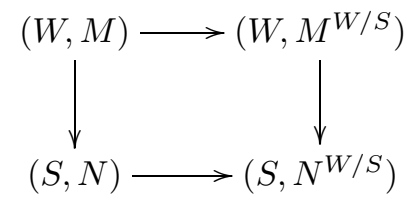

that is, the logarithmic data simply corresponds to a morphism of log structures $t^{W / S}: N^{W / S} \rightarrow N$. We will reserve the notation $t^{W / S}$ to always indicate this morphism and denote it by $t$ to simplify notation when no confusion may arise.

- There is a chart for the morphism $t^{W / S}: N^{W / S} \rightarrow N$ of the form

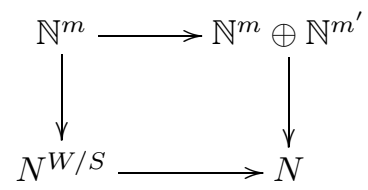


Here the top map is of the form $(d, 0)$, where $d=\left(d_{1}, \cdots, d_{m}\right)$ is a diagonal matrix of natural numbers.

From now on we will refer to log FM type spaces of X simply as log FM spaces and to extended log twisted FM type spaces of $\mathrm{X}$ as extended log twisted FM spaces for brevity. Having defined both the sources and the targets of log stable maps, we can now give the definition of the central objects of study of this paper.

Definition 2.5. A log stable map from a log curve $(C, M) /(S, N)$ to an extended $\log$ twisted FM space $(W, K) /(S, N)$ is a log morphism $f:(C, M) \rightarrow(W, K)$ over $(S, N)$ such that, over each point $s \in S$ :

- The cokernel of the map $N_{s}^{W / S} \rightarrow N_{s}$ has rank equal to the number of non-distinguished nodes.

- The map $f^{*} K_{s} \rightarrow M_{s}$ is simple at the distinguished nodes.

- Stability: The automorphism group $\operatorname{Aut}\left(f_{s}\right)$ is finite.

- The following minimality condition holds: Either $(C, M) /(S, N)$ is a minimal log curve or, if not, then there exists a subsheaf of monoids $N^{\prime}$ of $N$, which is a locally free $\log$ structure on $S$, such that $N=\mathbb{N} \oplus N^{\prime}, N^{C / S} \rightarrow N^{\prime}$ minimal, and $N^{W / S} \rightarrow \mathbb{N}$ surjective.

We explain the terminology: Over each $s \in S, C_{s}$ is a nodal curve and $W_{s}$ is an extended $\log$ twisted FM space. A node of $C_{s}$ is called distinguished if it maps into the singular locus of $W_{s}$ and is non-distinguished otherwise. A morphism between locally free log structures is called simple if it is given by a diagonal matrix, as in the definition of extended log twisted FM spaces above. An automorphism of $f:(C, M) \rightarrow(W, K)$ over $(S, N)$ is a cartesian diagram over $(S, N)$

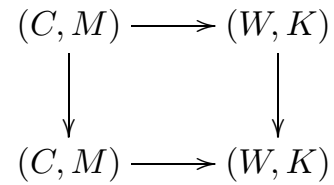

that respects the map to $X$; that is, on the level of underlying schemes we have

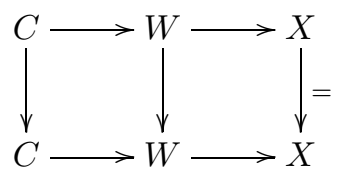

Remark 2.1. The minimality condition slightly deviates from Kim's definition. In Kim10, the definition of a log stable map requires that the log curve $(C, M) \rightarrow$ $(S, N)$ be minimal. This neglects the possibility that there is no node in the curve $C$ mapping to the original divisor $D=D[0]$. The typical example of this situation is in $(X, D)=\left(\mathbb{P}^{2}, \mathbb{P}^{1}\right)$, where a line $\mathbb{P}^{1}$ rotates to collapse into $D$ : the limit map is the map from $\mathbb{P}^{1}$ to $X[1]$ that sends all of $\mathbb{P}^{1}$ into $X[1]-X$, with the image line intersecting $D[1]$ transversely. There is a more satisfying, intrinsic explanation of the minimality condition. Minimality is a categorical property: Minimal log schemes $(S, N)$ are precisely the log schemes one must restrict to in order to be able to consider a stack over $\log$ schemes, such as $\mathcal{M}_{g, n}^{\log }$ or the stack of all $\log$ maps into extended log twisted FM spaces, as a log stack. This is the content of the paper Gil11]. Kim's minimal log curves are precisely the minimal objects for the stack $\mathcal{M}_{g, n}^{\log }$. However, the minimal objects for the stack of all log maps into 
extended log twisted FM spaces that satisfy the first three properties include the case that no node of the curve maps into $D[0]$, and we must include this case in the definition. Here we also remark that what we call minimal here is the same thing as what is called basic in Che14, AC11, GS11. Both notions correspond to the same categorical notion of minimality.

Let us describe the logarithmic data concretely in the case when $S=\operatorname{Spec} k$ is a geometric point. This description will be useful in what follows. The locally free $\log$ structures on $S$ are free in this case, described by a chart $\mathbb{N}^{r}$ for certain integers $r$; specifically, we have

$$
\begin{aligned}
N^{C / S} & =\mathbb{N}^{m^{\prime \prime}} \oplus \mathbb{N}^{m^{\prime}} \oplus k^{*}, \\
N^{W / S} & =\mathbb{N}^{m} \oplus k^{*}, \\
N & =\mathbb{N}^{m} \oplus \mathbb{N}^{m^{\prime}} \oplus k^{*} .
\end{aligned}
$$

The morphisms $s^{C / S}:=s, t^{W / S}:=t$ are described on the level of characteristic monoids as follows: $\bar{t}: \bar{N}^{W / S} \rightarrow \bar{N}$ is given by a diagonal matrix of the form $\left(d_{1}, \cdots, d_{m}, 0, \cdots, 0\right)$, as above. The morphism $\bar{s}: \bar{N}^{C / S} \rightarrow \bar{N}$ is given by a matrix of the form $(\Gamma, \mathrm{id})$, where $\Gamma$ is a generalized diagonal matrix:

$$
\left(\begin{array}{cccc}
\Gamma_{1,1} \cdots \Gamma_{1, k_{1}} & 0 \cdots 0 & \cdots & 0 \cdots 0 \\
0 \cdots 0 & \Gamma_{2,1} \cdots \Gamma_{2, k_{2}} & \cdots & 0 \cdots 0 \\
\vdots & \vdots & \ddots & \vdots \\
0 \cdots 0 & 0 \cdots 0 & \cdots & \Gamma_{m, 1} \cdots \Gamma_{m, k_{m}}
\end{array}\right)
$$

The integers $k_{1}, \cdots, k_{m}$ add up to $m^{\prime \prime}$. It is possible that the first row of the matrix is 0 , in which case $d_{1}=1$. This happens when no node of the curve maps to the original divisor $D=D[0]$. Otherwise, the log curve $(C, M) \rightarrow(S, N)$ is minimal, which means that there is no common divisor between the integers $\Gamma_{i, k_{1}}, \cdots, \Gamma_{i, k_{i}}$. For each $\Gamma_{i, j}$, there is an integer such that $d_{i}=\Gamma_{i, j} l_{i, j}$. In other words, there is a commutative diagram

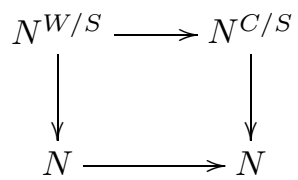

We will now fix a stack $\mathcal{B}$ of certain $\log$ FM spaces and denote by $\mathcal{B}^{\text {etw }}$ the stack whose objects are extended log twisted FM spaces whose underlying spaces are in $\mathcal{B}$. We denote by $\mathcal{U}$ and $\mathcal{U}^{\text {etw }}$ the universal family of $\mathcal{B}$ and $\mathcal{B}^{\text {etw }}$ respectively. In other words, we consider spaces $W \in \mathcal{B}$ but endow them with log structures as above. We will consider the stack $\mathcal{M}_{\Gamma}^{\log }(\mathcal{U} / \mathcal{B})$ of log stable maps to targets in $\mathcal{B}^{\text {etw }}$. It is proven Kim10] that if the stack $\mathcal{B}$ is algebraic, $\mathcal{M}_{\Gamma}^{\log }(\mathcal{U} / \mathcal{B})$ is also algebraic.

Remark 2.2. Let us at this point explain the connection with Jun Li's original definitions and clarify this concept geometrically. A family of expansions $W \rightarrow S$ of a pair $(X, D)$ or, similarly, of a $D$-semistable degeneration $X=Y_{1} \cup_{D} Y_{2}$ has canonical log structures that determine log FM spaces. The canonical log structure on a family of expansions is obtained in a manner formally identical to the way that the canonical log structure on a nodal curve is obtained. A detailed treatment of the canonical log structures on expansions can be found in Olsson's paper Ols03. 
Briefly, there is an algebraic stack $\mathcal{B}$ parametrizing expansions. The stack $\mathcal{B}$ is in fact the open substack of the stack $\mathfrak{M}_{0,3}$ of 3 marked, genus 0 prestable curves where the first two markings are on the first component of the curve and the third marking on the last; see for instance GV05. In $\mathcal{B}$ there is a normal crossings divisor corresponding to singular expansions; therefore, $\mathcal{B}$ admits a log structure $\mathcal{M}_{\mathcal{B}}$. Similarly, the universal family $\mathcal{U}$ over $\mathcal{B}$ admits a log structure $\mathcal{M}_{\mathcal{U}}$. A family of expansions corresponds to a cartesian diagram

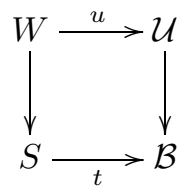

The pullback $\log$ structures $t^{*} \mathcal{M}_{\mathcal{B}}$ and $u^{*} \mathcal{M}_{\mathcal{U}}$ on $S$ and $W$ are what we denoted by $N^{W / S}$ and $M^{W / S}$ above. Therefore, expansions are examples of log FM spaces. We may thus consider log stable maps to expansions. The underlying morphism of schemes of such a log stable map is a relative stable map in the sense of Jun $\mathrm{Li}$; the predeformability condition is enforced by the requirement that the map is a map of $\log$ schemes. The log structures are thus additional algebraic data on a relative stable map. The log structures encode essential geometric information very conveniently. Suppose for simplicity that $S=\operatorname{Spec} k$ is a geometric point. We have seen above the form of the $\log$ structures $N^{C / S}, N^{W / S}, N$, and the maps between them. The rank of $N^{W / S}$, which we denoted by the number $m$ above, indicates that the target is the $m$-th expansion $(X[m], D[m])$ of $(X, D)$. The number $m^{\prime}$ is the number of non-distinguished nodes. The number $m^{\prime \prime}$ is the number of distinguished nodes. The matrix $\Gamma$ above indicates that $k_{1}$ of the distinguished nodes map to the first singular locus in $\left(X[m], D[m]\right.$ ) (namely to $D[0]$ ), $k_{2}$ map to $D[1]$, and so forth. The contact order of the $j$-th node mapping to the $i$-th singular locus is $l_{i, j}$. Note that once the underlying stable map is fixed, the diagram

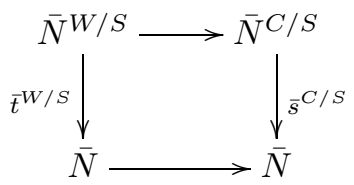

between the characteristic monoids of the log structures is determined. This means that in order to determine the full diagram

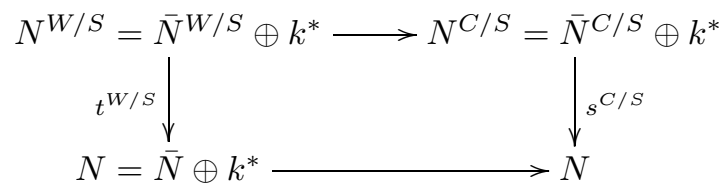

we need to determine the elements of $k^{*}$ to which the generators of $N^{C / S}$ and $N^{W / S}$ are mapping. In fact, all generators of $N^{W / S}$ may be chosen to map into $1 \in k^{*}$ after automorphism, so it is enough to treat only $N^{C / S}$. Matrix 1 in Remark 2.1 indicates that $s^{C / S}$ has the following form:

$$
e_{i j} \mapsto\left(\Gamma_{i j} e_{i}, u_{i j}\right)
$$


Note however that the units $u_{i j}$ are restricted: they must satisfy the equation $u^{l_{i j}}=1$, in order for the diagram

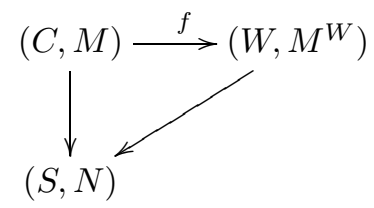

to commute. This shows that there is a finite number of ways to give to a relative stable map the structure of a log stable map. In other words, if $\mathcal{M}_{\Gamma}(X, D)$ denotes Jun Li's space of expansions and $\mathcal{M}_{\Gamma}^{\log }(\mathcal{U} / \mathcal{B})$ Kim's space of log stable maps to expansions of $(X, D)$, which is algebraic stack since the stack of expansions $\mathcal{B}$ is algebraic, there is a forgetful morphism

$$
\mathcal{M}_{\Gamma}^{\log }(\mathcal{U} / \mathcal{B}) \rightarrow \mathcal{M}_{\Gamma}(X, D)
$$

For the rigorous definition of the morphism $\pi^{b}: \mathcal{M}_{\Gamma}^{\log }(\mathcal{U} / \mathcal{B}) \rightarrow \mathcal{M}_{\Gamma}(X, D)$ we refer to the work of Gross and Siebert GS11 and the paper AMW12 of Abramovich, Marcus, and Wise. This is the left vertical arrow of diagram $*$ of the introduction. The fact that relative stable maps are a locally closed substack of the stack of all maps expresses the fact that the predeformability condition is locally closed. The fact that the stack of log stable maps is open in the stack of all log maps expresses the fact that predeformability is enforced by requiring that the map from a nodal curve to an expansion be a log map.

\section{EQUivariant EMBEDDING}

Since Kim's moduli space does not carry a fundamental class but rather a virtual fundamental class, in order to prove a localization formula we need to use the virtual localization formula of Graber-Pandharipande GP99. Traditionally, to use the results of [GP99, one needs to establish the following technical condition.

Theorem 3.1. There is a locally closed equivariant immersion of $\mathcal{M}_{\Gamma}^{\log }(\mathcal{U} / \mathcal{B})$ into a smooth Deligne-Mumford stack.

In fact, after the results of Chang-Kiem-Li in CKL17, this is no longer necessary. We nevertheless prove the theorem, as we believe that the result and its proof are of independent interest. We will do this by proving that $\mathcal{M}_{\Gamma}^{\log }(\mathcal{U} / \mathcal{B})$ satisfies a slightly stronger condition, also shared by Jun Li's space $\mathcal{M}_{\Gamma}(X, D)$. Namely we will prove that $\mathcal{M}_{\Gamma}^{\log }(\mathcal{U} / \mathcal{B})$ satisfies the following property, which we will abbreviate as property $\mathrm{SE}$ (for strong embedding property):

- $\mathcal{M}_{\Gamma}^{\log }(\mathcal{U} / \mathcal{B})=[V / G]$ is a global quotient, where $G$ is a reductive group and $V$ is a locally closed subset of a smooth projective $W$ with an action of $G$ extending that of $V$.

- There is a $\mathbb{C}^{*} \times G$ action on $W$ which preserves $\mathrm{V}$ and descends to the $\mathbb{C}^{*}$ action on $[V / G]$.

In GV05, it is shown that $\mathcal{M}_{\Gamma}(X, D)$ satisfies SE by an explicit construction. We have seen there is a morphism $\mathcal{M}_{\Gamma}^{\log }(\mathcal{U} / \mathcal{B}) \rightarrow \mathcal{M}_{\Gamma}(X, D)$; this morphism is in fact finite, as shown in Lemma 3.2 below. Therefore, it suffices to show the following lemma; the idea of the proof is due to Vistoli. 
Lemma 3.1. Suppose $f: X \rightarrow Y$ is a $\mathbb{C}^{*}$ equivariant finite morphism between Deligne-Mumford stacks, and assume that $Y$ satisfies $S E$. Then $X$ satisfies $S E$ as well and thus embeds $\mathbb{C}^{*}$ equivariantly into a smooth Deligne-Mumford stack.

Proof. Since $Y$ satisfies SE, we may write $Y=[V / G] \subset[W / G]$ with $V \subset W$ a $\mathbb{C}^{*} \times G$ equivariant locally closed subset and $W$ a smooth projective variety. We can find a $\mathbb{C}^{*} \times G$ equivariant open smooth subvariety $W_{o} \subset W$ such that:

(1) $G$ acts on $W_{0}$ with reduced finite stabilizers,

(2) $V$ is a closed subvariety of $W_{0}$.

(Note that this is clearly possible since $[V / G]$ is Deligne-Mumford by the hypothesis, or, equivalently, $G$ acts on $V$ with finite reduced stabilizers). The composed morphism $f: X \rightarrow Y \rightarrow\left[W_{o} / G\right]$ is then still $\mathbb{C}^{*}$ equivariant and finite, so by replacing $V$ with $W_{o}$ we may assume $V$ is smooth. Since the morphism $X \rightarrow Y$ is $\mathbb{C}^{*}$ equivariant, we obtain a morphism $\bar{f}:\left[X / \mathbb{C}^{*}\right] \rightarrow\left[Y / \mathbb{C}^{*}\right]$. Here $\left[X / \mathbb{C}^{*}\right]$ denotes the stack quotient in the sense of Romagny Rom05, though our notation is slightly different. Assume for a moment that $\left[Y / \mathbb{C}^{*}\right]$ has the resolution property ([Tot04]), i.e., that any coherent sheaf on $\left[Y / \mathbb{C}^{*}\right]$ admits a surjective morphism from a locally free sheaf on $\left[Y / \mathbb{C}^{*}\right]$. Then, the sheaf $\bar{f}_{*}\left(\mathcal{O}_{\left[X / \mathbb{C}^{*}\right]}\right)$, which is coherent since $f$ is finite, is the quotient of a locally free sheaf $\mathcal{E}$ on $\left[Y / \mathbb{C}^{*}\right]$. In other words, $\left[X / \mathbb{C}^{*}\right]$ embeds into a vector bundle over $\left[Y / \mathbb{C}^{*}\right]$. That is, there is a $\mathbb{C}^{*}$ equivariant morphism of $X$ into a $\mathbb{C}^{*}$ equivariant vector bundle over $Y=[V / G]$, hence a $\mathbb{C}^{*}$ equivariant embedding of $X$ into a smooth DM stack. In particular, $X$ admits a $\mathbb{C}^{*}$ equivariant embedding into a stack of the form $[U / G]$, where $U$ is a smooth vector bundle over $V$, so it is, in fact, a quotient. To see that $X$ satisfies $\mathrm{SE}$, then, the only thing that remains to be shown is that $U$ embeds $\mathbb{C}^{*} \times G$ equivariantly into a smooth and projective variety as a locally closed subset. We give the proof after proving the resolution property for $\left[Y / \mathbb{C}^{*}\right]$, as it requires essentially the same argument.

We thus show that $\left[Y / \mathbb{C}^{*}\right]=\left[[V / G] / \mathbb{C}^{*}\right]$ does, indeed, have the resolution property. Equivalently, we prove that any $\mathbb{C}^{*}$ equivariant coherent sheaf $\mathcal{F}$ on $[V / G]$ admits a $\mathbb{C}^{*}$ equivariant surjection from a $\mathbb{C}^{*}$ equivariant locally free sheaf.

Let $p: V \rightarrow[V / G]$ be the projection. Since we assumed above that $V$ is smooth and quasiprojective, by [MFK94, Corollary 1.6], we can find a $G \times \mathbb{C}^{*}$-equivariant immersion $i: V \rightarrow \mathbb{P}^{n}$. Let $\mathcal{O}_{V}(1)=i^{*} \mathcal{O}_{\mathbb{P}^{n}}(1)$ and consider a $\mathbb{C}^{*}$ equivariant coherent sheaf $\mathcal{F}$ on $[V / G]$. Since the $G \times \mathbb{C}^{*}$ action on $V$ descends to the $\mathbb{C}^{*}$ action on the quotient $[V / G]$, the pullback $p^{*} \mathcal{F}$ is $G \times \mathbb{C}^{*}$ equivariant. It is also coherent. Therefore, since $V$ is quasiprojective, we may pick a large $N$ so that the twisted sheaf $p^{*} \mathcal{F}(N)$ is generated by global sections, say $s_{1}, s_{2}, \cdots, s_{m} \in H^{0}\left(V, p^{*} \mathcal{F}(N)\right)$. Let $V_{1}=\left\langle s_{1}, s_{2}, \ldots, s_{m}\right\rangle$ be their linear span in $H^{0}\left(V, p^{*} \mathcal{F}(N)\right)$. Following the argument in the proof of [MFK94, pp. 25-26, Lemma*], we can find

$$
V_{1} \subset V_{2} \subset H^{0}\left(V, p^{*} \mathcal{F}(N)\right),
$$

where $V_{2}$ is a $\mathbb{C}^{*} \times G$ equivariant finitely generated subspace. We therefore obtain a natural $\mathbb{C}^{*} \times G$ equivariant surjection of sheaves on $V$

$$
V_{2} \otimes \mathcal{O}_{V} \rightarrow p^{*} \mathcal{F}(N)
$$

hence a natural $\mathbb{C}^{*} \times G$ equivariant surjection

$$
V_{2}(-N) \rightarrow p^{*} \mathcal{F}
$$


which descends to a $\mathbb{C}^{*}$ equivariant surjection from a $\mathbb{C}^{*}$ equivariant locally free sheaf on $[V / G]$ to $\mathcal{F}$. This shows that $\left[Y / \mathbb{C}^{*}\right]$ has the resolution property. Note that this argument also suffices to complete the proof that $X$ satisfies SE: we have already seen that $X$ embeds $\mathbb{C}^{*}$ equivariantly into a vector bundle $[U / G]$, where $\mathrm{U}$ is a vector bundle over $\mathrm{V}$; let $U=\operatorname{Spec}\left(S\left(\mathcal{E}^{\prime}\right)\right)$ for some locally free sheaf $\mathcal{E}^{\prime}$ on $V$. Then the argument just given shows that $\mathcal{E}^{\prime}$ admits a $\mathbb{C}^{*} \times G$ equivariant surjection $\left(\mathcal{O}_{V}(-M)\right)^{k} \rightarrow \mathcal{E}^{\prime}$ for some integers $M, k$. Therefore, $[U / G]$ admits a $\mathbb{C}^{*}$ equivariant locally closed immersion to the quotient of the projectivized bundle $\mathbb{P}\left(\mathcal{O}_{\mathbb{P}^{n}}(-M)^{k} \oplus \mathcal{O}_{\mathbb{P}^{n}}\right)$ over $\mathbb{P}^{n}$ (i.e., a smooth projective variety) by $G$.

Lemma 3.2. The morphism $\mathcal{M}_{\Gamma}^{\log }(\mathcal{U} / \mathcal{B}) \rightarrow \mathcal{M}_{\Gamma}(X, D)$ is finite.

Proof. We need to show that every geometric point of $\mathcal{M}_{\Gamma}(X, D)$, that is, every relative stable map $f$, has a finite number of preimages and that the morphism is representable: for each preimage $f$, the map $\operatorname{Aut}(f) \rightarrow \operatorname{Aut}(f)$ is injective. The discussion in section 2, or Remark 6.3.1 in [Kim10, establishes the finiteness of the preimages. Then let $f$ be a log stable map lying over $f$. Denote the base by $(S, N)$, where $S=$ Spec $k$, the source curve by $C$, and the target by $W=X\left[m^{\prime \prime}\right]$. Let $m, m^{\prime}$ be the number of distinguished and non-distinguished nodes of $f$ respectively, and let $m^{\prime \prime}$ be the number of nodes of $W$. We have $N^{C / S}=\mathbb{N}^{m} \oplus \mathbb{N}^{m^{\prime}} \oplus k^{*}, N^{W / S}=$ $\mathbb{N}^{m^{\prime \prime}} \oplus k^{*}$, and $N=\mathbb{N}^{m^{\prime \prime}} \oplus \mathbb{N}^{m^{\prime}} \oplus k^{*}$. An automorphism of $f$ lying over the identity automorphism of $f$ is simply an automorphism of the logarithmic structures, that is, an automorphism $A$ of $N$ that respects the maps $s:=s^{C / S}: N^{C / S} \rightarrow N$, $t:=t^{W / S}: N^{W / S} \rightarrow N$. In other words, we are looking for commutative diagrams

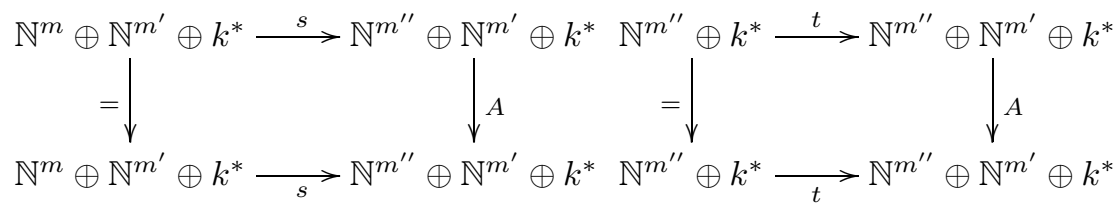

The induced matrix $\bar{s}$ on the level of characteristic monoids is a generalized diagonal matrix which is the identity on the last $m^{\prime}$ components, and similarly $\bar{t}$ is a diagonal matrix with finite cokernel on the first $m$ components. Therefore $\bar{A}$ must be a diagonal matrix as well, in fact, the identity on characteristic monoids. It follows that each factor of $\mathbb{N}^{m^{\prime \prime}}$ contributes to automorphisms separately, and we may thus assume $m^{\prime \prime}=1$ without loss of generality. In other words, the automorphism group of $N$ splits as a product of the automorphism groups contributed by each node of the target. We are thus reduced to studying three cases: (a) either there are $m$ distinguished nodes mapping to the node of $W$; (b) there is a node in $W$ but no node in $C$, i.e., $m=m^{\prime}=0$ (cf. the minimality condition of Definition 2.5); or (c) there is one non-distinguished node and no distinguished node, i.e., $m=0, m^{\prime}=1$, since every non-distinguished node contributes precisely one factor of $\mathbb{N}$ in $N$. The first case is most interesting. In this case, the two diagrams take the form

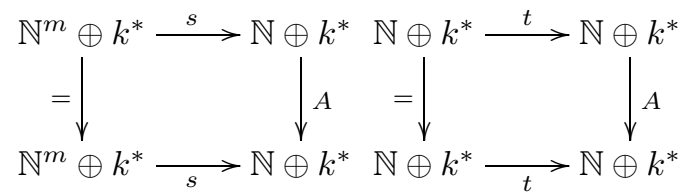


These are homomorphisms of log structures, thus lie over the map to the field $k$, and the factor of $k^{*}$ maps identically to itself. The automorphism $A$ is determined by its action on the generator $e$ of $\mathbb{N}$ and thus takes the form $e \mapsto(e, v)$ for a unit $v \in k^{*}$. On the other hand, we have seen in formula (1) that

$$
e_{i} \mapsto\left(\Gamma_{i} e, u_{i}\right),
$$

and thus commutativity of the diagram implies $\left(\Gamma_{i} e, u_{i} v^{\Gamma_{i}}\right)=\left(\Gamma_{i} e, u_{i}\right)$; that is, $v^{\Gamma_{i}}=1$ for all $i$. By minimality, the greatest common divisor of the $\Gamma_{i}$ is 1 , and thus $v=1$.

In the case (b), the diagrams become

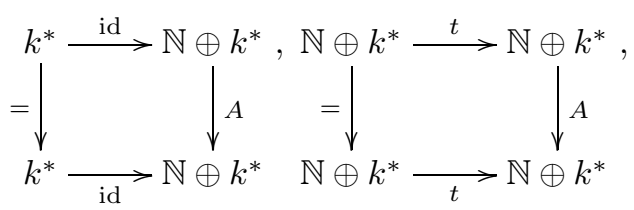

and the map $t$ is of the form $e \mapsto(d e, 1)$. However, minimality requires that $d=1$. Hence, $A$, which has the form $e \mapsto(e, v)$, must have $v=1$ and is thus trivial. Finally, in case (c) the diagrams become

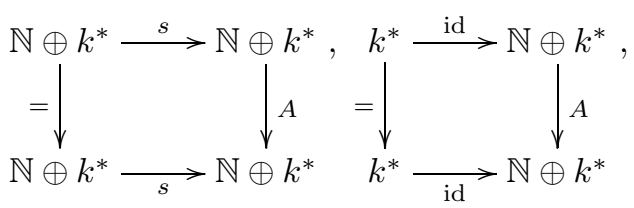

and $s$ is the identity on the level of characteristics, so $A$ is trivial as well, by the same argument as in (b).

From the two lemmas it follows that the localization formula of GP99 can be applied to $\mathcal{M}_{\Gamma}^{\log }(\mathcal{U} / \mathcal{B})$.

\section{The OBSTRUCTION THEORY}

In this section we analyze the obstruction theory of $\mathcal{M}_{\Gamma}^{\log }(\mathcal{U} / \mathcal{B})$. We will write $M$ for $\mathcal{M}_{\Gamma}^{\log }(\mathcal{U} / \mathcal{B})$ to ease the notation. There is a forgetful morphism, $\tau: M \rightarrow \mathcal{M B}$, where

$$
\mathcal{M B}=\mathfrak{M}_{g, n}^{\log } \times_{\text {LOG }} \mathcal{B}^{\text {etw }}
$$

Here, the category LOG is the category whose objects are log schemes and morphisms are strict log morphisms. The stack $\mathcal{M B}$ parametrizes, over a scheme $S$, pairs $(C, M) /(S, N)$ and $(W, K) /(S, N)$ of an $n$-marked log curve over $(S, N)$ and an FM space in $\mathcal{B}^{\text {etw }}$ over the same $\log$ scheme $(S, N)$. The morphism $\tau$ sends a $\log$ stable map to the pair consisting of the source of the map and the target; it is the forgetful morphism forgetting the data of the map. The stack $\mathcal{M B}$ is the analogue of the Artin stack $\mathfrak{M}$ of prestable curves in ordinary Gromov-Witten theory. It is also smooth and, in fact, log smooth, as it further has the structure of a log stack.

By standard properties of the cotangent complex, the morphism $\tau$ induces a distinguished triangle

$$
\tau^{-1} \mathcal{L}_{\mathcal{M B}} \rightarrow \mathcal{L}_{M} \rightarrow \mathcal{L}_{M / \mathcal{M B}} \rightarrow \tau^{-1} \mathcal{L}_{\mathcal{M B}}[1]
$$


Consider the diagram

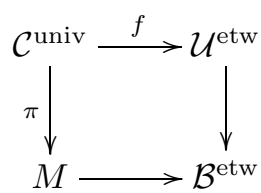

Here $\mathcal{C}^{\text {univ }}$ is the universal family of $M$ and $\mathcal{U}^{\text {etw }}$ the universal family over $\mathcal{B}^{\text {etw }}$. The morphism $f$ is the evaluation map and $\pi$ the projection. It is proven in section 7 of Kim10 that there is a canonical morphism

$$
E^{\bullet}=\left(R \pi_{*} f^{*} T_{\mathcal{U}^{\text {etw }} / \mathcal{B}^{\text {etw }}}^{\log }\right)^{\vee} \rightarrow \mathcal{L}_{M / \mathcal{M B}}^{\log }
$$

which is a (relative) perfect obstruction theory. The morphism $\tau$ is strict; therefore the log cotangent complex $\mathcal{L}_{M / \mathcal{M B}}^{\log }$ coincides with the ordinary cotangent complex $\mathcal{L}_{M / \mathcal{M B}}$. Furthermore, the stack $\mathcal{M B}$ is smooth; therefore $\mathcal{L}_{\mathcal{M B}}$ is a two-term complex concentrated in degrees 0 and 1 (it is an Artin stack, so it has automorphisms). We therefore have a diagram

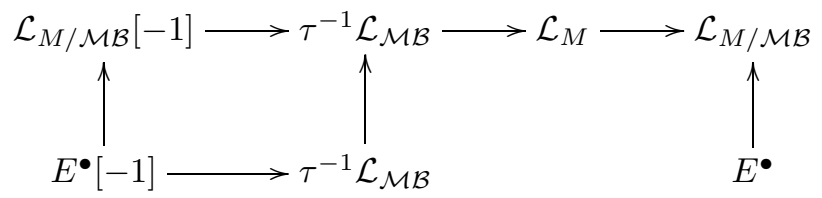

We may fill in the lower row by the cone of $E^{\bullet}[-1] \rightarrow \tau^{-1} \mathcal{L}_{\mathcal{M B}}$ to obtain an (absolute) perfect obstruction theory for $M$. We denote the tangent and obstruction sheaves on $M$ by $\mathcal{T}^{1}, \mathcal{T}^{2}$ respectively. Applying the functor $R \operatorname{Hom}\left(\bullet, \mathcal{O}_{M}\right)$, we obtain the following lemma.

Lemma 4.1. Over a geometric point $f:\left(C, M_{C}\right) /(k, N) \rightarrow\left(W, M_{W}\right) /(k, N)$, the tangent space $\mathcal{T}_{f}^{1}$ and obstruction space $\mathcal{T}_{f}^{2}$ of $M$ fit into an exact sequence

$$
0 \rightarrow \mathfrak{a u t}(\tau(f)) \rightarrow H^{0}\left(f^{*} T_{W}^{\mathrm{log}}\right) \rightarrow \mathcal{T}_{f}^{1} \rightarrow \operatorname{Def}(\tau(f)) \rightarrow H^{1}\left(f^{*} T_{W}^{\log }\right) \rightarrow \mathcal{T}_{f}^{2} \rightarrow 0 .
$$

The term $T_{W}^{\log }$ denotes the logarithmic tangent sheaf, that is, the dual of the sheaf of relative logarithmic differentials of the logarithmic map $\left(W, M_{W}\right) \rightarrow(k, N)$ [Kat, section 1.7]. The term aut $(\tau(f))$ refers to the group of first order infinitesimal automorphisms of $\tau(f)$. To carry out localization calculations, we need to know the equivariant Euler classes of $\mathcal{T}^{1}, \mathcal{T}^{2}$. Since the Euler class is a K-theory invariant, it is enough to understand the other four terms in the exact sequence. The terms $H^{k}\left(f^{*} T_{W}^{\mathrm{log}}\right), k=0,1$, are the cohomology groups of explicit locally free sheaves on the curve $C$, which may be calculated by hand. In fact, more can be said:

Lemma 4.2. Suppose $\pi: W \rightarrow X$ denotes the canonical contraction map. Then $T_{W}^{\log }=\pi^{*} T_{X}^{\log }=\pi^{*} T_{X}(-\log D)$.

Proof. We recall the explicit construction of the log schemes $X[n]$. For details of the construction, the reader is referred to [Li01]. Set $Y[0]=X[0]=X$; let $Y[1]$ be the blowup of $Y[0] \times \mathbb{A}^{1}=X \times \mathbb{A}^{1}$ along the divisor $D \times 0$, with a divisor $D[1] \subset Y[1]$ defined as the proper transform of $D \times \mathbb{A}^{1}$. We view $Y[1]$ as a family of log schemes over $\mathbb{A}^{1}$, with logarithmic structure on $\mathbb{A}^{1}$ coming from the divisor $0 \in \mathbb{A}^{1}$, the product $\log$ structure on $X \times \mathbb{A}^{1}$, and the natural log structure on the blowup $Y[1]$. The $\log$ scheme $X[1]$ over Spec $k$ is the fiber of this family over 0 , with the induced 
logarithmic structures on $X[1]$ and the base Spec $k$. Next, $Y[2]$ is the blowup of $Y[1] \times \mathbb{A}^{1}$ along $D[1] \times 0$, with a divisor $D[2]$ the proper tranform of $D[1] \times \mathbb{A}^{1}$. This is viewed as a family over $\mathbb{A}^{2}$, with the standard toric log structure on $\mathbb{A}^{2}$ coming from the axes, and the log structure on $Y[2]$ again arising from the product log structure on $Y[1] \times \mathbb{A}^{1}$ and blowing up. The scheme $X[2]$ is the fiber over $0 \in \mathbb{A}^{2}$. In general, $Y[n]$ is constructed from $Y[n-1] \times \mathbb{A}^{1}$ by blowing up $D[n-1] \times 0$; this is viewed as a family over $\mathbb{A}^{n}$ with the toric logarithmic structure on $\mathbb{A}^{n}$ and the logarithmic structure on $Y[n]$ coming from $Y[n-1] \times \mathbb{A}^{1}$ and blowing up; $D[n]$ is the proper transform of $D[n-1] \times \mathbb{A}^{1}$; and $X[n]$ is the fiber over $0 \in \mathbb{A}^{n}$. Then, for each $i=0,1, \ldots, n$, the ideal sheaf of $D[i] \times 0$ in $Y[i] \times \mathbb{A}^{1}$ is actually a logarithmic ideal sheaf, so the resulting morphism $\pi: Y[n] \rightarrow X \times \mathbb{A}^{n}$ in the diagram

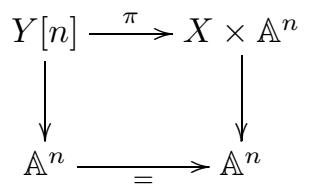

is a sequence of logarithmic blowups in the sense of [Kat, 1.3] (see also, for example, [Niz06, section 4] for the definition of the logarithmic blowup). Since logarithmic blowups are $\log$ étale morphisms, $\pi$ is $\log$ étale. It follows that the log tangent bundle of $Y[n]$ over $\mathbb{A}^{n}$ is the pullback of the log tangent bundle of $X \times \mathbb{A}^{n}$ over $\mathbb{A}^{n}$, which is simply the log tangent bundle of $X$. Since log étale morphisms are stable under base change, it follows that the tangent bundle of $X[n]$ over the base Spec $k$ is pulled back from $X$ as well, as claimed.

Thus the two terms $H^{k}\left(f^{*} T_{W}^{\mathrm{log}}\right)$ only depend on the logarithmic map from $C$ to $X$. What we have to understand are the two terms $\mathfrak{a u t}(\tau(f)), \operatorname{Def}(\tau(f))$, that is, the infinitesimal automorphism group and tangent space of a point of $\mathcal{M B}$. In the discussion that follows, we restrict attention to the stack $\mathcal{M B}$ when $\mathcal{B}$ is the stack of expansions of $X$, discussed in Remark 2.2.

We will understand the deformation group in terms of the stack of twisted stable curves $\mathfrak{M}_{g, n}^{\mathrm{tw}}$ of Abramovich-Vistoli [AV02, which is well understood. To do so, we must digress a bit. First, it will be easier for technical reasons to compare the deformation theory of $\mathcal{M B}$ with the deformation theory of the stack of log twisted curves of Olsson Ols07, which we denote by $\mathfrak{M}_{g, n}^{\operatorname{logtw}}$. It is shown in Ols07] that $\mathfrak{M}_{g, n}^{\text {logtw }} \cong \mathfrak{M}_{g, n}^{\mathrm{tw}}$. Recall the definition of $\mathfrak{M}_{g, n}^{\log t \mathrm{w}}$.

Definition 4.1. A log twisted curve over a scheme $S$ is a $\log$ curve $C \rightarrow S, N^{C / S} \stackrel{\alpha}{\rightarrow}$ $N$, where $N$ is locally free and $\alpha$ is an injection that is locally given by a diagonal matrix.

Let us fix some notation. Denote the set of non-distinguished nodes of the curve by $R$, the set of distinguished nodes by $S$, and the nodes of the target by $T$. In the notation above, we would have $|T|=m,|R|=m^{\prime},|S|=m^{\prime \prime}$. Furthermore, let

$$
\mathcal{A}=\left[\mathbb{A}^{1} / \mathbb{G}_{m}\right]
$$

denote the "universal target": this is the moduli space that over a scheme $S$ parametrizes line bundles $L$ over $S$, together with a section $s \in \Gamma(S, L)$, up to isomorphism. 
Consider now a family $F$ of log stable maps over a scheme $S$, which specializes over a geometric point Spec $k$ to a map $f:\left(\left(C, M_{C}\right) /(k, N), \vec{x}\right) \rightarrow\left(W, M_{W}\right) /(k, N)$. We must now define several morphisms.

First, consider the image of $F$ in $\mathfrak{M}_{g, n}^{\log }$ under the natural forgetful morphism, which forgets the data of the target and the map. Étale locally around $\left(C, M_{C}\right) /(k, N)$, we have a map $\mathfrak{M}_{g, n}^{\log } \rightarrow \mathfrak{M}_{g, n}^{\text {logtw }}$. The morphism is defined as follows: for an étale neighborhood $\left(C^{\prime}, M_{C^{\prime}}\right) /\left(S^{\prime}, N\right)$ of $\left(C, M_{C}\right) /(k, N)$ in which all $\log$ structures $N^{C^{\prime} / S^{\prime}}$ and $N$ are actually free, the map $s^{C^{\prime} / S^{\prime}}: N^{C^{\prime} / S^{\prime}} \rightarrow N$

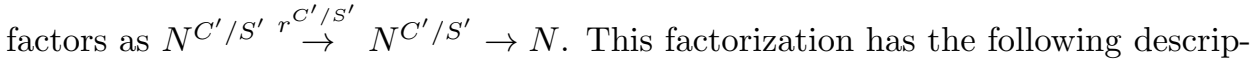
tion on the level of characteristic monoids: we have seen in formula (11) of section 2 that the morphism $\bar{N}^{C^{\prime} / S^{\prime}}=\mathbb{N}^{m^{\prime \prime}} \oplus \mathbb{N}^{m^{\prime}} \rightarrow \bar{N}=\mathbb{N}^{m} \oplus \mathbb{N}^{m^{\prime}}$ has the form ( $\Gamma$,id), with $\Gamma$ a generalized diagonal matrix. This factors as

$$
\mathbb{N}^{m^{\prime \prime}} \oplus \mathbb{N}^{m^{\prime}} \stackrel{(\gamma, \text { id })}{\rightarrow} \mathbb{N}^{m^{\prime \prime}} \oplus \mathbb{N}^{m^{\prime}} \stackrel{p}{\rightarrow} \mathbb{N}^{m} \oplus \mathbb{N}^{m^{\prime}}
$$

where $\gamma$ is the matrix $\Gamma$ 'made diagonal', i.e.,

$$
\gamma=\left(\begin{array}{cccccccc}
\Gamma_{1,1} & 0 & 0 & \ldots & 0 & 0 & \ldots & 0 \\
0 & \Gamma_{1,2} & 0 & \ldots & 0 & 0 & \ldots & 0 \\
\vdots & \vdots & \ddots & \vdots & \vdots & \vdots & \vdots & \vdots \\
0 & 0 & \ldots & \Gamma_{1, k_{1}} & 0 & \ldots & \ldots & 0 \\
0 & 0 & \ldots & 0 & \Gamma_{2,1} & 0 & \cdots & 0 \\
\vdots & \vdots & \vdots & \vdots & \vdots & \ddots & \vdots & \vdots \\
0 & \cdots & \cdots & \cdots & \cdots & \cdots & \cdots & \Gamma_{m, k_{m}}
\end{array}\right)
$$

and the map $p$ is the projection that sends the first $k_{1}$ coordinates of $\mathbb{N}^{m^{\prime \prime}}$ to the first coordinate of $\mathbb{N}^{m}$, the next $k_{2}$ coordinates to the second coordinate of $\mathbb{N}^{m}$, and so forth. It remains to explain how the morphism lifts from the level of characteristic monoids to the actual log structures; this is the evident extension of formula (1') of Remark 2.2. If $s^{C^{\prime} / S^{\prime}}$ maps

$$
e_{i j} \mapsto\left(\Gamma_{i j} e_{i}, u_{i j}\right),
$$

then we now have that $r^{C^{\prime} / S^{\prime}}$ maps

$$
e_{i j} \mapsto\left(\Gamma_{i j} e_{i j}, u_{i j}\right) .
$$

Therefore, from the data $\left(C^{\prime} \rightarrow S^{\prime}, N^{C^{\prime} / S^{\prime}} \rightarrow N\right)$ we obtain a log twisted curve $\left(C^{\prime} \rightarrow S^{\prime}, r^{C^{\prime} / S^{\prime}}: N^{C^{\prime} / S^{\prime}} \rightarrow N^{C^{\prime} / S^{\prime}}\right)$. This defines the required morphism. When we compose with the isomorphism $\mathfrak{M}_{g, n}^{\text {logtw }} \cong \mathfrak{M}_{g, n}^{\mathrm{tw}}$, the twisted curve $\mathcal{C}$ we obtain is the curve $C$ with the $j$-th node of $C$ mapping to the $i$-th node of $W$ twisted by $\Gamma_{i j}$.

Next, in an étale neighborhood $\mathfrak{M}_{f}$ of the image of $f$ in $\mathfrak{M}_{g, n}^{\operatorname{logtw}}$, we have a morphism $\mathfrak{M}_{f} \rightarrow \mathcal{A}^{m^{\prime \prime}}$, which can be described as follows. The $m^{\prime \prime}$ nodes signify that in an étale neighborhood of the image of $f$ in $\mathfrak{M}_{g, n}^{\text {logtw }}$, the image of $f$ is in the intersection of $m^{\prime \prime}$ boundary divisors of $\mathfrak{M}_{g, n}^{\operatorname{logtw}}$ intersecting transversally. In an étale neighborhood $\mathfrak{M}_{f}$ of the image of $f$ the $m^{\prime \prime}$ divisors thus determine $m^{\prime \prime}$ line bundles with sections and thus yield the desired map to $\mathcal{A}^{m^{\prime \prime}}$. We may further obtain a morphism $\mathcal{B}_{f} \rightarrow \mathcal{A}^{m}$ from an étale neighborhood of $f$ in $\mathcal{B}^{\text {etw }}$ in a similar fashion. 
Putting everything together, we obtain an étale neighborhood $\mathcal{M} \mathcal{B}_{f}$ of $\tau(f)$ in $\mathcal{M B}$ and a morphism $\phi$ :

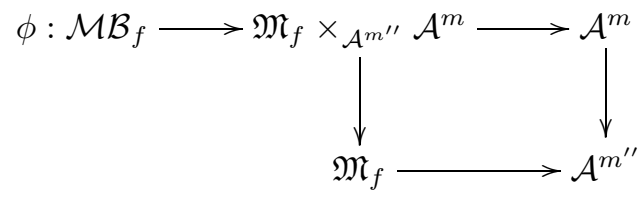

Let us choose a point $\tau(f)^{\prime}$ in $\mathcal{M B}_{f}$ lying over $\tau(f)$.

Lemma 4.3. The morphism $\phi$ is étale at $\tau(f)^{\prime}$.

Proof. Since all stacks in question are smooth, it is sufficient to show that their tangent spaces at $\tau(f)^{\prime}$ and $\phi\left(\tau(f)^{\prime}\right)$ respectively are isomorphic. It will be clear from the proof that we may reduce to the case where two distinguished nodes map into a single node of the target, that is, where $m^{\prime \prime}=2, m=1, m^{\prime}=0$. The argument for this is the same as the argument in the proof of Lemma 3.2. We will restrict attention to this case to simplify the notation. We are therefore given étale locally around $\tau(f)$ a diagram

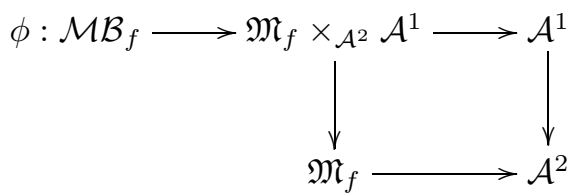

and we want to show that $\phi$ induces an isomorphism of tangent spaces. Since étale maps between smooth stacks induce isomorphisms on tangent spaces, we will work directly with the original moduli stacks and study their tangent spaces around the respective images of the point $f$ rather than their étale neighborhoods, again in order to keep the notation simple. Recall that the element $\tau(f) \in \mathcal{M B}$ (Spec $k$ ) consists of data of a pair $(C / \operatorname{Spec} k, W=X[1] / \operatorname{Spec} k)$ and two diagrams of log structures

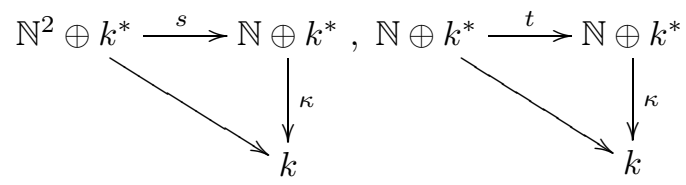

Here the maps $s$ and $t$ are the maps $s^{C / \text { Spec } k}, t^{W / \text { Spec } k}$ respectively. We have by formula (1?) that $e_{i} \mapsto\left(\Gamma_{i} e, u_{i}\right)$ (the $\Gamma_{i}$ here are what we would have called $\Gamma_{1 i}$ above, but since there is only one target node, we drop the first index to simplify notation). The map $t$ is given by mapping the generator $e \rightarrow(d e, 1)$, and the rest of the arrows send the generators of $\mathbb{N}, \mathbb{N}^{2}$ to 0 in $k$.

On the other hand, an element of $\mathfrak{M}_{g, n}^{\text {logtw }} \times \mathcal{A}^{2} \mathcal{A}^{1}$ (Spec $k$ ) corresponds to a triple $(x, y, \alpha)$ of an element $x$ of $\mathfrak{M}_{g, n}^{\text {logtw }}(\operatorname{Spec} k)$, an element $y \in \mathcal{A}^{1}(\operatorname{Spec} k)$, and an isomorphism between their images in $\mathcal{A}^{2}(\operatorname{Spec} k)$. The element $x$ corresponds to a 
pair of a nodal curve $C /$ Spec $k$ as above and a diagram of log structures

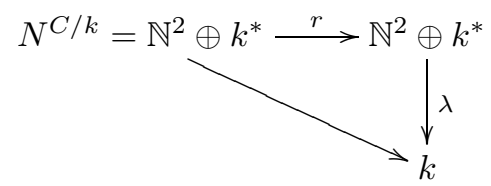

where the top map $r=r^{C / \text { Spec } k}$ is an injection $e_{i} \mapsto\left(\Gamma_{i} e_{i}, u_{i}\right)$; the map $\lambda$ sends $e_{i} \mapsto a_{i} \in k$; and the diagonal map is determined by commutativity, $e_{i} \mapsto u_{i} a_{i}^{\Gamma_{i}}$. An element of $\mathcal{A}^{1}($ Spec $k)$ is a line bundle over Spec $k$ together with a section, in other words, an element $a \in \mathbb{A}^{1}$. An element of $\mathcal{A}^{2}$ (Spec $k$ ) is similarly a pair $\left(a_{1}, a_{2}\right) \in \mathbb{A}^{2}$. The map $\mathcal{A}^{1}(\operatorname{Spec} k) \rightarrow \mathcal{A}^{2}(\operatorname{Spec} k)$ sends $a \mapsto(a, a)$, and the map $\mathfrak{M}_{g, n}^{\text {logtw }}(\operatorname{Spec} k) \rightarrow \mathcal{A}^{2}(\operatorname{Spec} k)$ sends the data just described to the pair $\left(a_{1}, a_{2}\right)$. Therefore the triple $(x, y, \alpha)$ has $x$ as above, $y=a \in \mathbb{A}^{1}$, and $\alpha=\left(c_{1}, c_{2}\right) \in\left(k^{*}\right)^{2}$, an isomorphism of $(a, a)$ with $\left(a_{1}, a_{2}\right)$ in $\mathbb{A}^{2}$; that is,

- $c_{i}=\frac{a_{i}}{a}$ if all $a$ and $a_{i}$ are non-zero,

- $c_{i}$ is arbitrary if $a=a_{1}=a_{2}=0$.

The morphism $\phi: \mathcal{M B}_{f} \rightarrow \mathfrak{M}_{f} \times \mathcal{A}^{2} \mathcal{A}^{1}$ then sends the data corresponding to $\tau(f)$ to the triple $(x, 0,(1,1))$, where $x$ is the curve $(C /$ Spec $k)$ and the diagram (3) has $r, \beta$ determined by $r\left(e_{i}\right)=\left(\Gamma_{i} e_{i}, u_{i}\right)$ and $\beta\left(e_{i}\right)=0$, with $\Gamma_{i}, u_{i}$ as in the definition of $s$.

To show that $\phi$ induces an isomorphism of tangent spaces we consider isomorphism classes of morphisms from Spec $k[\epsilon]$ to all stacks in question extending the given data over Spec $k$. A morphism Spec $k[\epsilon] \rightarrow \mathfrak{M}_{g, n}^{\text {logtw }}$ corresponds to a pair of an infinitesimal deformation $C^{\prime} / \operatorname{Spec} k[\epsilon]$ of $C$ and a diagram

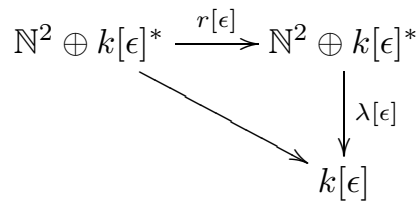

lying over (3). Therefore, we must have $e_{i} \rightarrow\left(\Gamma_{i} e_{i}, u_{i}+v_{i} \epsilon\right)$ under $r[\epsilon] ; \lambda[\epsilon]$ maps $e_{i} \rightarrow \alpha_{i} \epsilon$. The diagonal arrow is determined by commutativity $e_{i} \mapsto\left(\alpha_{i}\right)^{\Gamma_{i}}\left(u_{i}+v_{i} \epsilon\right)$.

Morphisms Spec $k[\epsilon] \rightarrow \mathcal{A}^{1}$ and Spec $k[\epsilon] \rightarrow \mathcal{A}^{2}$ lying over the given elements $0 \in$ $\mathcal{A}^{1}(\operatorname{Spec} k),(0,0) \in \mathcal{A}^{2}($ Spec $k)$ are again line bundles over Spec $k[\epsilon]$, which are thus trivial, together with a section restricting to 0 over Spec $k$; hence they correspond to $\alpha \epsilon,\left(\alpha_{1} \epsilon, \alpha_{2} \epsilon\right)$ respectively. Under the morphism $\mathfrak{M}_{g, n}^{\operatorname{logtw}}(k[\epsilon]) \rightarrow \mathcal{A}^{2}(\operatorname{Spec} k[\epsilon])$ maps the extension of $x$ to the pair $\left(\alpha_{1} \epsilon, \alpha_{2} \epsilon\right)$, where the $\alpha_{i}$ are the ones appearing in diagram (4). Isomorphisms between $(\alpha \epsilon, \alpha \epsilon)$ and $\left(\alpha_{1} \epsilon, \alpha_{2} \epsilon\right)$ restricting to $(1,1)$ over Spec $k$ are of the form $\left(1+\beta_{1} \epsilon, 1+\beta_{2} \epsilon\right)$. Note that $\beta_{1}, \beta_{2}$ can be arbitrary; however, in order for an isomorphism to exist, the condition $\alpha_{1}=\alpha_{2}=\alpha$ is forced. Therefore, the choices involved in extending $(x, y, \alpha)$ are the choices of the deformation $C^{\prime}$ of $C$ and the numbers $\alpha, v_{i}, \beta_{i}$. Notice however that the choice of either the $v_{i}$ or the $\beta_{i}$ can be eliminated via an isomorphism, for consider two pairs 
$\left(1+\beta_{1} \epsilon, 1+\beta_{2} \epsilon\right)$ and $\left.\left(1+\beta_{1}^{\prime} \epsilon, 1+\beta_{2}^{\prime} \epsilon\right)\right)$. There is an isomorphism

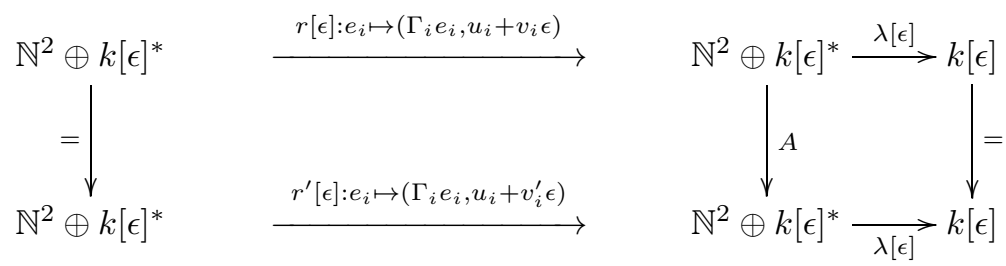

with the vertical arrow being the isomorphism $A: e_{i} \mapsto\left(e_{i}, 1+c_{i} \epsilon\right)$ where $c_{i}=$ $\beta_{i}-\beta_{i}^{\prime}$ and $v_{i}^{\prime}=v_{i}+u_{i} \Gamma_{i} c_{i}$. In other words, if we denote by $x[\epsilon]$ the extension of $x$ determined by choosing $r[\epsilon]$ as the extension of $r$ (that is, with the choice of the unit $v_{i}$ ) and by $x[\epsilon]^{\prime}$ the one with $r^{\prime}[\epsilon]$ as the extension of $r$ (with the unit $\left.v_{i}^{\prime}\right)$ we have an isomorphism between the triple $\left(x[\epsilon], \alpha \epsilon,\left(1+\beta_{1} \epsilon, 1+\beta_{2} \epsilon\right)\right)$ and $\left(x[\epsilon]^{\prime}, \alpha \epsilon,\left(1+\beta_{1}^{\prime} \epsilon, 1+\beta_{2}^{\prime} \epsilon\right)\right)$.

To summarize, the choice of the extension of the image of $\tau(f)$ in $\mathfrak{M}_{g, n}^{\text {logtw }}$ corresponds to the data of a choice of a deformation $C^{\prime}$ of $C$ and the choices of the numbers $\alpha, v_{i}$.

On the other hand, a choice of an extension of $\tau(f)$ in $\mathcal{M B}$ corresponds to the data of a deformation $C^{\prime}$ of $C$, a deformation $W^{\prime}$ of $W$ in $\mathcal{B}$, which is necessarily trivial, and diagrams

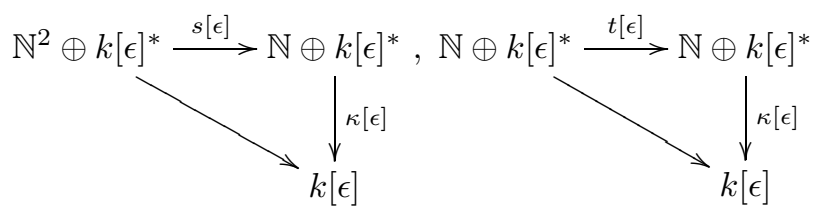

lying over (2). The extension $s[\epsilon]$ maps $e_{i} \mapsto\left(\Gamma_{i} e, u_{i}+v_{i} \epsilon\right), t[\epsilon]$ maps $e \mapsto(d e, 1+v \epsilon)$, $\kappa[\epsilon]$ maps $e \mapsto \alpha \epsilon$, and the diagonal arrows are determined by commutativity. Notice that up to isomorphism, there is only one choice for the right diagram, the choice of the number $\alpha$. Again, this is because from the diagram

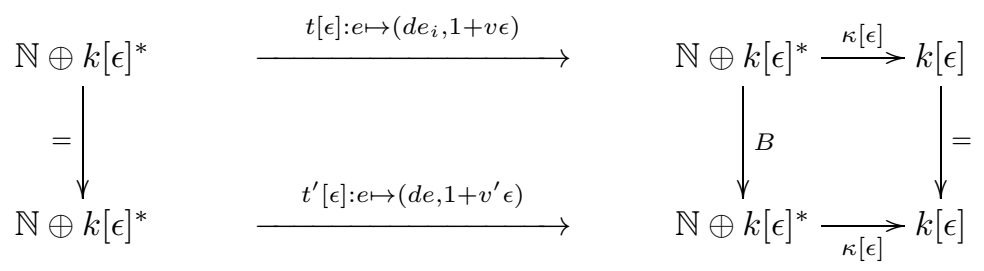

with $B$ the isomorphism $e \mapsto(e, 1+c \epsilon)$, and $v^{\prime}=v+c d \epsilon$ we get an isomorphism between the extension determined by the extensions $s[\epsilon], \kappa[\epsilon]$ of $s, \kappa$ with the extension determined by $s^{\prime}[\epsilon], \kappa[\epsilon]$. Since by varying $c$ the expression $v+c d \epsilon$ varies through all elements of $k$, the choice of $s[\epsilon]$ is eliminated up to isomorphism, as claimed. Once the isomorphism $B$ is fixed, though, the diagrams on the left with different choices of $v_{i}$ remain distinct. In other words, the choices involved in extending $\tau(f)$ are up to isomorphism the extension $C^{\prime}$ of $C$ and the numbers $\alpha, v_{i}$. These are precisely the same choices as involved in extending $(x, 0,(1,1))$. This concludes the proof of the lemma.

Remark 4.1. The geometric meaning of the number $\alpha_{i}$ in the map $\mathbb{N}^{2} \oplus k[\epsilon]^{*} \rightarrow$ $k[\epsilon], e_{i} \mapsto \alpha_{i} \epsilon$ is that the $i$-th node is smoothed with speed $\alpha_{i}$ in the moduli space 
of twisted curves. The geometric significance of Lemma 4.3 then is that in $\mathcal{M B}$, all nodes mapping to the same node of the target must be smoothed simultaneously, with the same speed: the speed with which the node of the target is being smoothed.

The lemma in particular implies that we may calculate $\operatorname{Def}(\tau(f))$ as follows: Let us write $\sum x_{i}$ for the divisor of marked points and $\mathcal{C}$ the twisted curve obtained from $f$ as explained. The tangent space to the stack of twisted curves is given by the ext group

$$
\operatorname{Ext}^{1}\left(\Omega_{\mathcal{C}}\left(\sum x_{i}\right), \mathcal{O}_{\mathcal{C}}\right)
$$

where $\mathcal{O}_{\mathcal{C}}$ and $\Omega_{\mathcal{C}}$ are the structure sheaf and sheaf of Kahler differentials of the twisted curve respectively. The "local-to-global" spectral sequence for Ext says that the tangent space fits into the short exact sequence

$$
\begin{aligned}
0 \rightarrow H^{1}\left(\operatorname{Hom}\left(\Omega_{\mathcal{C}}\left(\sum x_{i}\right), \mathcal{O}_{\mathcal{C}}\right)\right) & \rightarrow \operatorname{Ext}^{1}\left(\Omega_{\mathcal{C}}\left(\sum x_{i}\right), \mathcal{O}_{\mathcal{C}}\right) \\
& \rightarrow H^{0}\left(\operatorname{Ext}^{1}\left(\Omega_{\mathcal{C}}\left(\sum x_{i}\right), \mathcal{O}_{\mathcal{C}}\right)\right) \rightarrow 0 .
\end{aligned}
$$

Here Hom and Ext are underlined to indicate that we are taking the sheaf Hom and Ext respectively. The rightmost group in the exact sequence has a canonical description as follows. Let $R, S, T$ denote the set of non-distinguished nodes of the curve, the set of distinguished nodes of the curve, and the set of nodes of the target, as above. Furthermore, given a node $x$ in the curve $\mathcal{C}$, let $\mathcal{C}_{x}^{i}, i=1,2$, denote the two components of $\mathcal{C}$ at $x$. Then we have

$$
H^{0}\left(\operatorname{Ext}^{1}\left(\Omega_{\mathcal{C}}\left(\sum x_{i}\right), \mathcal{O}_{\mathcal{C}}\right)\right) \cong \bigoplus_{\text {nodes of } \mathrm{C}} T_{x} \mathcal{C}_{x}^{1} \otimes T_{x} \mathcal{C}_{x}^{2} \cong \mathbb{C}^{m^{\prime}+m^{\prime \prime}}
$$

There is a diagonal map $\mathbb{C}^{m} \rightarrow \mathbb{C}^{m^{\prime \prime}}$ which simply sends the coordinate $e_{y}$ corresponding to a node $y \in T$ to the sum of the coordinates corresponding to the nodes in $\mathcal{C}$ mapping to $y$, that is, $\sum_{x ; f(x)=y} e_{x}$. This is in fact the map of tangent spaces of the map $\mathcal{A}^{m} \rightarrow \mathcal{A}^{m^{\prime \prime}}$ described above. Just as the tensor product $\bigoplus_{\text {nodes of } \mathrm{C}} T_{x} \mathcal{C}_{x}^{1} \otimes T_{x} \mathcal{C}_{x}^{2}$ describes intrinsically the part of the deformations of the curve that smooth the nodes, the group $\bigoplus_{i=1}^{n} H^{0}\left(\mathcal{W}, N_{\mathcal{D}[i] / \mathcal{X}[i-1]} \otimes N_{\mathcal{D}[i] / \mathcal{X}[i]}\right)$ describes the part of the deformations of $\mathcal{W}=\mathcal{X}[n]$ that smooth the nodes of $\mathcal{W}$. Here, $\mathcal{W}$ is obtained from $W$ by twisting along the divisor $D[i]$ by the integer $d_{i}$ via the map $t^{W / S}: N^{W / S} \rightarrow N$, just as $\mathcal{C}$ is obtained from $C$ via the map $r^{C / S}$. Then, the fiber diagram of Lemma 4.3 implies the following.

Corollary 4.1. The tangent space $\operatorname{Def}(\tau(f))$ to $\mathcal{M B}$ is the fiber product

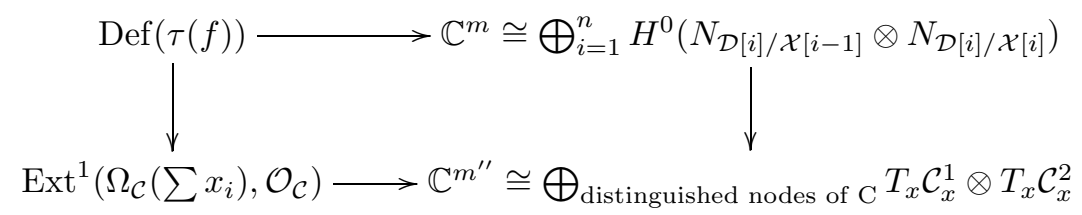

The proof of Lemma 4.3 also allows us to understand the automorphism group $\mathfrak{a} \mathfrak{u t}(\tau(f))$.

Corollary 4.2. An infinitesimal automorphism of $f$ in $\mathcal{M B}$ consists of an infinitesimal automorphism of the source curve $C$ fixing the marked points and an 
infinitesimal automorphism of $W$ in $\mathcal{B}$ :

$$
\mathfrak{a u t}(\tau(f))=\mathfrak{a u t}(C, \vec{x}) \oplus \mathfrak{a u t}_{\mathcal{B}}(W)=\mathfrak{a u t}(C, \vec{x}) \oplus \mathbb{C}^{m} .
$$

Proof. We give the details for the case when two nodes of $C$ map into a node of $W$, as in Lemma 4.3. The general case reduces to this as in the proof of Lemma 3.2. The group of infinitesimal automorphisms of $\tau(f)$ consists of the group of automorphisms of the trivial extension of $\tau(f)$ over $k[\epsilon]$ lying over the identity automorphism of $\tau(f)$. This is an infinitesimal automorphism of $C$, an infinitesimal automorphism of $W$ in $\mathcal{B}$, which consists of a copy of $\mathbb{C}$ for each expanded component of $W$, and an automorphism $A$ of the $\log$ structure $N=\mathbb{N} \oplus k[\epsilon]^{*}$ which lies over the identity automorphism over $\operatorname{Spec} k$ and which is compatible with both diagrams

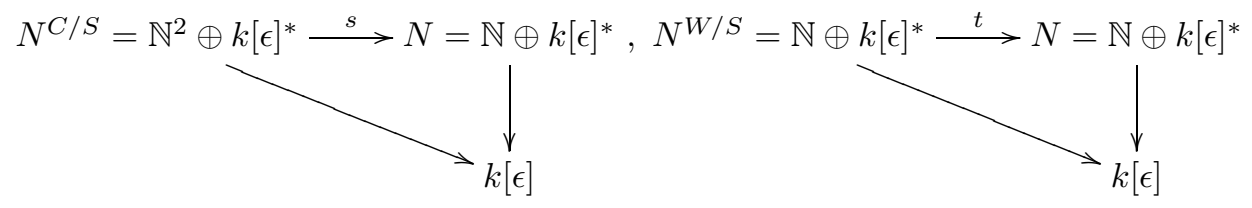

Here $s\left(e_{i}\right)=\left(\Gamma_{i} e, u_{i}\right), t(e)=(d e, 1)$ as always. An automorphism $A$ of $N$ must map the generator of $\mathbb{N} e \mapsto(e,(1+c \epsilon))$ for some $c \in k$ if it is to reduce to the identity over Spec $k$. In order for the automorphism to be compatible with the second diagram, that is, in order for

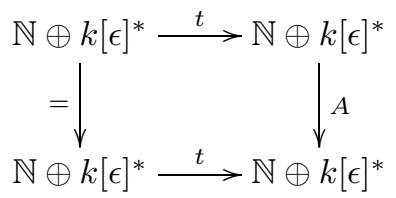

to commute, we must have $A t(e)=\left(d e,(1+c \epsilon)^{d}\right)=(d e, 1+c d \epsilon)=(d e, 1)=t(e)$, which implies that $c=0$. So the logarithmic structures contribute no infinitesimal automorphisms.

\section{The VIRTUAL LOCALIZATION FORMUla}

We are now in a position to derive the virtual localization formula for $\mathcal{M}_{\Gamma}^{\log }(\mathcal{U} / \mathcal{B})$ in the case when the pair $(X, D)$ carries a $T=\mathbb{C}^{*}$-action leaving $D$ pointwise fixed. The ideas of this section can essentialy be found in the paper of Graber-Vakil GV05. For the convenience of the reader, we recall the form of relative virtual localization formulas and refer the reader to the paper of Graber-Pandharipande GP99 for details.

5.1. Graber-Pandharipande virtual localization. Suppose $M$ is a DM stack with a $\mathbb{C}^{*}$-action equipped with a $\mathbb{C}^{*}$-equivariant perfect obstruction theory $C^{\bullet} \rightarrow$ $\mathcal{L}_{M}$. Let $F_{a}$ denote the connected components of the fixed locus of $M$, which we refer to as the fixed loci for brevity, and denote the natural inclusion by $i_{a}: F_{a} \rightarrow$ $M$. The virtual localization formula reads

$$
\int_{[M]^{\mathrm{vir}}} \omega=\sum_{a} \int_{\left[F_{a}\right]^{\mathrm{vir}}} \frac{i_{a}^{*} \omega}{e^{T}\left(N_{F}^{\mathrm{vir}}\right)} .
$$

Here $\omega$ is a class in $A_{T}^{*}(M)$, the equivariant Chow ring of $M$ (or in equivariant cohomology). The integral $\int_{[M]^{\text {vir }}}$ is the proper pushforward map from $A_{T}^{*}(M) \rightarrow$ 
$A_{T}^{*}(p t)=A^{*}(B T) \cong \mathbb{C}[u]$. The term $e^{T}$ denotes the equivariant Euler class of a vector bundle, in this case, of the virtual normal bundle $N_{F_{a}}^{\mathrm{vir}}$ of $F_{a}$ in $M$. The qualification that the normal bundle is virtual means that we are not only taking the ordinary normal bundle in the tangent space but also keeping track of the obstruction bundle. More precisely, $N_{F}^{\text {vir }}$ is defined as $\mathcal{T}_{1}^{m}-\mathcal{T}_{2}^{m}$, the moving part of the tangent space minus the moving part of the obstruction space (the moving part of a representation is the subrepresentation where $T$ acts non-trivially). The Euler class of a sum of vector bundles is by definition the product of the Euler classes; the Euler class of the difference is thus the quotient. Finally, the virtual fundamental class of a fixed locus $[F]^{\text {vir }}$ is by definition the virtual fundamental class arising from the fixed part of the tangent/obstruction theory. Therefore, in order to give a localization formula we must identify the fixed loci and calculate the classes $e^{T}\left(N_{F}^{\mathrm{vir}}\right)$ for each of them.

5.2. Types of fixed loci. Consider a pair $(X, D)$ as above. This defines a stack of expanded targets $\mathcal{B}$, as explained in Remark 2.2. We fix discrete data $\Gamma=$ $\left(g, m, h, \vec{c}=\left(c_{1}, \cdots, c_{h}\right)\right)$, consisting of the genus of a curve, $m+h$ marked points, and the contact orders of the last $h$ marked points at the divisor at infinity $D[n]$ of the target $W=X[n]$. In this section we study the fixed loci of $\mathcal{M}_{\Gamma}^{\log }(\mathcal{U} / \mathcal{B})=M$. In the localization formula we will distinguish between two different types of fixed loci; the first type consists of morphisms to $X$ itself rather than an expansion of $X$. Such a locus is much simpler to understand than a general locus. The other type of fixed loci consist of those with targets $W=X[n]$ with $n>0$. The idea of the localization formula is to express the virtual fundamental classes of these loci recursively in terms of the simple loci and moduli spaces of log stable maps to the expanded part of $W$ only. We formalize this below.

Definition 5.1. A fixed locus $F \subset M$ is called simple if the general, hence every, element $f \in F$ has target $W=X[0]=X$. We denote a simple fixed locus with discrete data $\Gamma$ by $\mathcal{M}_{\Gamma}^{\log }(\mathcal{U} / \mathcal{B})^{\operatorname{sim}}=M^{\operatorname{sim}}$.

Similarly we define composite loci.

Definition 5.2. A fixed locus $F \subset M$ is called composite if the elements $f \in F$ map to targets $W=X[n]$ with $n>0$.

The simple loci $\mathcal{M}_{\Gamma}^{\log }(\mathcal{U} / \mathcal{B})^{\operatorname{sim}}$ are open substacks of $\mathcal{M}_{\Gamma}^{\log }(\mathcal{U} / \mathcal{B})$; therefore, all results of section 4 apply to the simple loci without change.

To understand composite loci, we need to understand log stable maps to the expanded part of $W=X[n]$, that is, to the scheme theoretic closure $Y=\overline{W \backslash X[0]}$ of the complement of the first component $X=X[0]$ in $X[n]$. The formal definition of such a log stable map is identical to the one given in Definition 2.5. The only differences in the theory of such maps arise from the fact that the rigidifying map to $X$ is much simpler, contracting all components to $D$. In the case of $X=\mathbb{P}^{1}$, which was the first case to be studied in the literature, the rigidifying map to $X$ becomes trivial. We thus call these maps unrigidified log stable maps. We denote the stack parametrizing the expanded part of expansions of $X$ by $\mathcal{B}^{\sim}$ and its universal family by $\mathcal{U}^{\sim}$. Similarly to $\mathcal{B}$, the stack $\mathcal{B}^{\sim}$ is isomorphic to the open substack of $\mathfrak{M}_{0,2}$ where the first marking is on the first component and the second marking is on the last component; see Remark 2.2. We will abusively denote the space of log stable 
maps to targets in $\mathcal{B}^{\sim}$ by $\mathcal{M}_{\Gamma}^{\log }(\mathcal{U} / \mathcal{B})^{\sim}$. The analogous stack of unrigidified relative stable maps $\mathcal{M}_{\Gamma}(X, D) \sim$ is introduced and studied in GV05.

Remark 5.1. The stack $\mathcal{M}_{\Gamma}^{\log }(\mathcal{U} / \mathcal{B})^{\sim}$ is very similar and often simpler than $\mathcal{M}_{\Gamma}^{\log }(\mathcal{U} / \mathcal{B})$. For instance, observe that

- The minimality condition in Definition 2.5 is actually simpler: it simply requires that the log curve $(C, M) \rightarrow(S, N)$ be minimal, since it is not possible to have a node of the target with no distinguished node mapping to it anymore.

- The analogous map $\mathcal{M}_{\Gamma}^{\log }(\mathcal{U} / \mathcal{B})^{\sim} \rightarrow \mathcal{M}_{\Gamma}(X, D)^{\sim}$ of Lemma 3.2 is also finite, as the map to $X$ is never required in the proof of Lemma 3.2.

- Theorem 3.1 applies without change. This is because it is shown in GV05. that $\mathcal{M}_{\Gamma}(X, D)^{\sim}$ satisfies the property SE, so Lemma 3.1 applies.

For the purposes of localization, we need to understand the deformation theory of $\mathcal{M}_{\Gamma}^{\log }(\mathcal{U} / \mathcal{B})^{\sim}$ carefully. This is essentially the same as that of $\mathcal{M}_{\Gamma}^{\log }(\mathcal{U} / \mathcal{B})$. Specifically, we introduce the analogue of the stack $\mathcal{M B}$,

$$
\mathcal{M B}^{\sim}=\mathfrak{M}_{g, n}^{\log } \times_{\mathrm{LOG}}\left(\mathcal{B}^{\sim}\right)^{\text {etw }},
$$

and its canonical morphism,

$$
\tau^{\sim}: \mathcal{M}_{\Gamma}^{\log }(\mathcal{U} / \mathcal{B})^{\sim} \rightarrow \mathcal{M B}^{\sim}
$$

The analogues of Lemma 4.1 and of Corollaries 4.1 and 4.2 are then as follows.

Lemma 5.1. Over a geometric point $f:\left(C, M_{C}\right) /(k, N) \rightarrow\left(Y, M_{Y}\right) /(k, N)$, the tangent space $\mathcal{T}_{f}^{1 \sim}$ and obstruction space $\mathcal{T}_{f}^{2 \sim}$ of $M^{\sim}$ fit into an exact sequence

$0 \rightarrow \mathfrak{a u t}\left(\tau^{\sim}(f)\right) \rightarrow H^{0}\left(f^{*} T_{Y}^{\log }\right) \rightarrow \mathcal{T}_{f}^{1 \sim} \rightarrow \operatorname{Def}\left(\tau^{\sim}(f)\right) \rightarrow H^{1}\left(f^{*} T_{Y}^{\log }\right) \rightarrow \mathcal{T}_{f}^{2 \sim} \rightarrow 0$.

Proof. The deformation theory of $\mathcal{M}_{\Gamma}^{\log }(\mathcal{U} / \mathcal{B})^{\sim}$ is the same as in section 4, induced by the relative perfect obstruction theory

$$
\left(E^{\sim}\right)^{\bullet}=\left(R \pi_{*} f^{*} T_{\mathcal{U}^{\sim \text { etw }} / \mathcal{B}^{\sim \text { etw }}}^{\log }\right)^{\vee} \rightarrow \mathcal{L}_{M \sim / \mathcal{M B}^{\sim}}^{\log }
$$

where $M$ has been replaced by $M^{\sim}$ and $\mathcal{B}$ by $\mathcal{B}^{\sim}$. This is true since Kim's proof works for any stack of log FM spaces, thus expansions and unrigidified expansions alike. Therefore, the discussion of section 4 applies as well, which results in the six-term exact sequence.

Furthermore, with the notation of Corollary 4.1, we have the following.

Corollary 5.1. The tangent space $\operatorname{Def}\left(\tau^{\sim}(f)\right)$ to $\mathcal{M B}$ is the fiber product

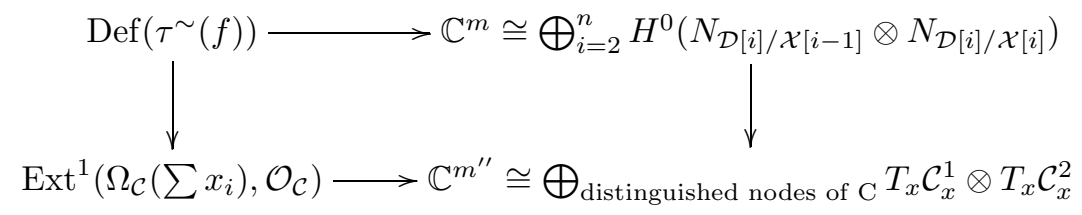

Proof. The corollary follows from Lemma 4.3, the lemma applies verbatim, as the map to $X$ is required nowhere in the proof. 
Finally,

Corollary 5.2. An infinitesimal automorphism of $f$ in $\mathcal{M B}^{\sim}$ consists of an infinitesimal automorphism of the source curve $C$ fixing the marked points and an automorphism of $W$ in $\mathcal{B}^{\sim}$ :

$$
\mathfrak{a u t}(\tau(f))=\mathfrak{a} \mathfrak{u t}(C, \vec{x}) \oplus \mathfrak{a} \mathfrak{u t} \mathfrak{B}_{\mathcal{B}}(W)=\mathfrak{a} \mathfrak{u t}(C, \vec{x}) \oplus \mathbb{C}^{m} .
$$

The two main differences between $\mathcal{M}_{\Gamma}^{\log }(\mathcal{U} / \mathcal{B})$ and $\mathcal{M}_{\Gamma}^{\log }(\mathcal{U} / \mathcal{B})^{\sim}$ are the following: First, the action of $T=\mathbb{C}^{*}$ on $\mathcal{M}_{\Gamma}^{\log }(\mathcal{U} / \mathcal{B})^{\sim}$ is trivial. This is because dilation of each component of $Y=\overline{W \backslash X[0]}$ by $c \in T$ is an automorphism of $Y$, and so the map $c f$ is isomorphic to $f$. Second, the log tangent bundle $T_{Y}^{\log }$ is trivial.

5.3. Description of the fixed loci in terms of known stacks. Consider now an element $f \in \mathcal{M}_{\Gamma}^{\log }(\mathcal{U} / \mathcal{B})$ in a composite fixed locus, mapping to a target $W=X[n]$. Define $C_{1}=f^{-1}(X), C_{2}=f^{-1}(Y)$ with $Y=\overline{W \backslash X[0]}$ as above, and let $f_{1}: C_{1} \rightarrow$ $X, f_{2}: C_{2} \rightarrow Y$ denote the restriction maps. The discrete data $\Gamma=(g, h, m, \vec{c})$ then splits into two sets of discrete data: $\Gamma_{1}=\left(g_{1}, \vec{\alpha}=\left(\alpha_{1}, \cdots, \alpha_{k}\right), S_{1}, \beta_{1}\right)$, consisting of the genus of $C_{1}$, a partition describing the behavior of $f_{1}$ over $D=D[0]$, the subset $S_{1}$ of the marked points on $C_{1}$ not mapping to $D$, and the homology class $\left(f_{1}\right)_{*}\left[C_{1}\right]$; and $\Gamma_{2}=\left(g_{2}, \vec{\alpha}, \vec{c}, S_{2}, \beta_{2}\right)$, consisting of the genus of $C_{2}$, the subset $S_{2}$ of the marked points on $C_{2}$ not mapping to $D$, the same partition $\vec{\alpha}$, the original partition $\vec{c}$ describing the behavior along the divisor at infinity, and the homology class determined by $f_{2}$. The data $\Gamma_{1}, \Gamma_{2}$ are locally constant on the fixed locus. Furthermore, $f_{1}$ and $f_{2}$ are naturally logarithmic stable maps, with logarithmic data determined only by $f$. Then, the map $f_{1}$ belongs to $\mathcal{M}_{\Gamma_{1}}^{\log }(\mathcal{U} / \mathcal{B})^{\text {sim }}$ and $f_{2}$ belongs to $\mathcal{M}_{\Gamma_{2}}^{\log }(\mathcal{U} / \mathcal{B})^{\sim}$. In what follows we will describe the substacks $F_{\Gamma_{1}, \Gamma_{2}}$ of the fixed locus of $\mathcal{M}_{\Gamma}^{\log }(\mathcal{U} / \mathcal{B})$, obtained by all possible splittings of the discrete data $\Gamma$ into $\left(\Gamma_{1}, \Gamma_{2}\right)$, in terms of known stacks involving $\mathcal{M}_{\Gamma_{1}}^{\log }(\mathcal{U} / \mathcal{B})^{\operatorname{sim}}$ and $\mathcal{M}_{\Gamma_{2}}^{\text {log }}(\mathcal{U} / \mathcal{B})^{\sim}$. To this end, we need to be able to glue any pair of log stable maps in $\mathcal{M}_{\Gamma_{1}}^{\log }(\mathcal{U} / \mathcal{B})^{\operatorname{sim}} \times$ $\mathcal{M}_{\Gamma_{2}}^{\log }(\mathcal{U} / \mathcal{B})^{\sim}$ into a log stable map in $\mathcal{M}_{\Gamma}^{\log }(\mathcal{U} / \mathcal{B})$. In order to do so, we need first of all to be able to glue the underlying maps, so we work right away with $\mathcal{M}_{\Gamma_{1}}^{\log }(\mathcal{U} / \mathcal{B})^{\operatorname{sim}} \times_{D^{k}} \mathcal{M}_{\Gamma_{2}}^{\log }(\mathcal{U} / \mathcal{B})^{\sim}$ and only need to glue the logarithmic data.

It is convenient to think about log structures in the Borne-Vistoli setting [BV12; thus, a $\log$ structure on a scheme $S$ is a sheaf of sharp monoids $M_{S}$ and a functor $F_{M_{S}}: \bar{M}_{S} \rightarrow \operatorname{Div}_{S}=\operatorname{Hom}\left(S, \mathcal{A}^{1}\right)=\operatorname{Hom}\left(S,\left[\mathbb{A}^{1} / \mathbb{G}_{m}\right]\right)$. Concretely, to every element of $\bar{M}_{S}(U)$ we assign a line bundle with a section on $U$ in a functorial manner. In our case everything is particularly simple, as the characteristic monoids $\bar{M}_{S}$ will be just of the form $\mathbb{N}^{k}$ for some integer $k$, as in fact the characteristic monoids of the relevant sublog structures will be constant, so all this data will amount to choosing $k$ line bundles with sections on $S$. A morphism between two $\log$ structures $M_{S}, N_{S}$ on $S$ is a map $\phi: \bar{M}_{S} \rightarrow \bar{N}_{S}$ such that $F_{N_{S}} \circ \phi$ is naturally isomorphic to $F_{M_{S}}$.

A. The canonical log structures on the glued source and target. Consider a family of nodal curves $p: C \rightarrow S$, for which $k$ nodes persist. We label the nodes (connected components of the relative singular locus) by $x_{1}, \cdots, x_{k}$, and by $C_{1}$ and $C_{2}$ the two components of the partial normalization of $C$ at the nodes. For notational convenience, we denote the images of the sections of $p_{1}: C_{1} \rightarrow S$ and 
$p_{2}: C_{2} \rightarrow S$ which map to the node $x_{i}$ by the same symbol. The canonical log structure $N^{C / S}$ on $S$ (see section 2) has a locally free sublog structure corresponding to the nodes. In the language of Borne-Vistoli the latter is the log structure $\left(\mathbb{N}^{k}, e_{i} \mapsto\left(\left(p_{\left.1\right|_{x_{i}}}\right)_{*}\left(N_{x_{i} / C_{1}}\right) \otimes\left(p_{\left.2\right|_{x_{i}}}\right)_{*}\left(N_{x_{i} / C_{2}}\right), 0\right)\right)$; that is, the line bundle that parametrizes deformations of the node in $C$ and with the 0 section, as $p: C \rightarrow S$ corresponds to a map to $\mathfrak{M}_{g, n}$ that maps into the boundary divisor determined by the node, and the log structure on $S$ is pulled back by the divisorial log structure on $\mathfrak{M}_{g, n}$ under this map. Similarly, for a family of singular expansions $q: W \rightarrow S$ in $\mathcal{B}$ for which the singularity persists, we will write $W=X_{1} \cup_{D_{S}} X_{2}$. Here $X_{1}=X \times S$ is the simple part of $W, X_{2} \in \mathcal{B}^{\sim}(S)$ its expanded part, and $D_{S}$ denotes the Cartier divisor in $X_{1}$ and $X_{2}$, canonically identified with $D \times S$ (see [Li01, section 4.1]), along which we glue them to obtain $W$. Since the fibers of $N_{D_{S} / X_{1}} \otimes N_{D_{S} / X_{2}}$ are trivial, a standard cohomology and base change argument shows that the pushforward of $N_{D_{S} / X_{1}} \otimes N_{D_{S} / X_{2}}$ via $D_{S} \rightarrow W \rightarrow S$ is locally free and that the adjunction $q_{\left.\right|_{D_{S}}}^{*} q_{\left.\right|_{D_{S}}}\left(N_{D_{S} / X_{1}} \otimes N_{D_{S} / X_{2}}\right) \rightarrow N_{D_{S} / X_{1}} \otimes N_{D_{S} / X_{2}}$ is an isomorphism (this can be checked on the local models of $W \rightarrow S$ described in [Li01]). Then the canonical $\log$ structure $N^{W / S}$ of $W$ on $S$ (see section 2) has a locally free sublog structure corresponding to $D_{S}$ given by $\left(\mathbb{N}, e \mapsto\left(q_{\left.\right|_{D_{S}}}\left(N_{D_{S} / X_{1}} \otimes N_{D_{S} / X_{2}}\right), t\right)\right)$, where $t=0$, since again the divisor persists.

B. Gluing the logarithmic maps. Suppose now that we have two families $f_{1}$ : $C_{1} \rightarrow X_{1}$ and $f_{2}: C_{2} \rightarrow X_{2}$ over $S$ in $\mathcal{M}_{\Gamma_{1}}^{\log }(\mathcal{U} / \mathcal{B})^{\text {sim }}$ and $\mathcal{M}_{\Gamma_{2}}^{\log }(\mathcal{U} / \mathcal{B})^{\sim}$ respectively. On the level of schemes, the problem has a unique gluing to a family

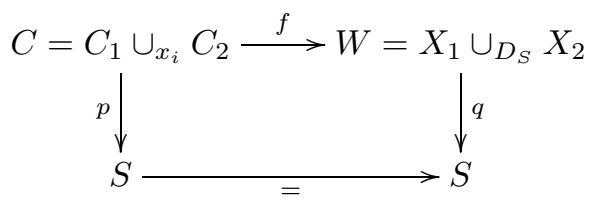

The map however is not a log map at the moment. To promote it to one, we must correct the $\log$ structure on $S$. Any $\log$ structure $M_{S}$ on $S$ that makes simultaneously $W$ and $C \log$ smooth over $S$ must receive maps from both canonical $\log$ structures $N^{C / S}$ and $N^{W / S}$. Furthermore, if $\alpha_{i}$ is the contact order of $f$ at $x_{i}$, the map $C \rightarrow W$ at $x_{i}$ must, at the level of $\log$ structures, have the form

$$
\begin{aligned}
M_{S} \oplus_{\mathbb{N}} \mathbb{N}^{2} \longrightarrow M_{S} \oplus_{\mathbb{N}} \mathbb{N}^{2} \\
e_{1} \mapsto \alpha_{i} e_{1} \\
e_{2} \mapsto \alpha_{i} e_{2} .
\end{aligned}
$$

Here, in the two pushouts, the map $\mathbb{N} \rightarrow M_{S}$ is the map determined from the maps $N^{W / S} \rightarrow M_{S}$ and $N^{C / S} \rightarrow M_{S}$ as the image of the generator corresponding to $D_{S} \subset W$ and $x_{i} \subset C$ respectively. The map $\mathbb{N} \rightarrow \mathbb{N}^{2}$ is the diagonal in both cases. Consequently, the image $m_{D}$ of the part of the log structure $N^{W / S}$ corresponding to $D_{S}$ in $M_{S}$ must be identified with $\alpha_{i}$ times the image $m_{i}$ of the part of the $\log$ structure $N^{C / S}$ corresponding to $x_{i}$ in $M_{S}$. We claim there is a log structure $N_{S}$ on $S$ with a unique map $N_{S} \rightarrow M_{S}$, i.e., an initial $\log$ structure that makes $f$ into a logarithmic stable map or, in other words, a minimal one (Definition 2.2). To see this, note that the maps $f_{1}, f_{2}$ restricted to $x_{i}$ give isomorphisms $f_{1}^{*} N_{D_{S} / X_{1}} \cong N_{x_{i} / C_{1}}^{\otimes \alpha_{i}}$ and $f_{2}^{*} N_{D_{S} / X_{2}} \cong N_{x_{i} / C_{2}}^{\otimes \alpha_{i}}$. Hence, pushing forward to $S$, we 
get isomorphisms

$$
\left(p_{\left.1\right|_{x_{i}}}\right)_{*}\left(f_{1}^{*}\left(N_{D_{S} / X_{1}}\right)\right) \otimes\left(p_{\left.2\right|_{x_{i}}}\right)_{*}\left(f_{2}^{*}\left(N_{D_{S} / X_{2}}\right)\right) \cong\left(p_{\left.1\right|_{x_{i}}}\right)_{*} N_{x_{i} / C_{1}}^{\otimes \alpha_{i}} \otimes\left(p_{\left.2\right|_{x_{i}}}\right)_{*} N_{x_{i} / C_{2}}^{\otimes \alpha_{i}} .
$$

Since $p_{1}$ and $p_{2}$ restricted to $x_{i}$ are isomorphisms, the notation can be simplified to

$$
f_{1}^{*}\left(N_{D_{S} / X_{1}}\right) \otimes f_{2}^{*}\left(N_{D_{S} / X_{2}}\right) \cong\left(N_{x_{i} / C_{1}} \otimes N_{x_{i} / C_{2}}\right)^{\otimes \alpha_{i}} .
$$

On the other hand, recall that $q_{\mid D_{S}}^{*} q_{\left.\right|_{D_{S}}}\left(N_{D_{S} / X_{1}} \otimes N_{D_{S} / X_{2}}\right) \cong N_{D_{S} / X_{1}} \otimes N_{D_{S} / X_{2}}$ (section 5.3.A). Since $q \circ f_{1}$ and $q \circ f_{2}$ restricted to $x_{i}$ are equal to $p_{1 \mid x_{i}}$ and $p_{2 \mid x_{i}}$ respectively we get simply that

$$
q_{\left.\right|_{D_{S}}}\left(N_{D_{S} / X_{1}} \otimes N_{D_{S} / X_{2}}\right) \cong\left(N_{x_{i} / C_{1}} \otimes N_{x_{i} / C_{2}}\right)^{\otimes \alpha_{i}} \text {. }
$$

Therefore, if $N_{S}$ is defined by

$$
\begin{array}{r}
\bar{N}_{S}=\mathbb{N}^{k} \oplus \mathbb{N} / \mathbb{N}\left(\alpha_{i} e_{i}-e\right) \\
e_{i} \mapsto\left(\left(N_{x_{i} / C_{1}} \otimes N_{x_{i} / C_{2}}\right), 0\right) \\
e \mapsto\left(q_{D_{D_{S}} *}\left(N_{D_{S} / X_{1}} \otimes N_{D_{S} / X_{2}}\right), 0\right)
\end{array}
$$

we see that there is a unique map $N_{S} \rightarrow M_{S}$ which sends $e_{i}$ to $m_{i}$ and $e$ to $m_{D}$.

This does not solve the problem still, as to produce a family in $\mathcal{M}_{\Gamma}^{\log }(\mathcal{U} / \mathcal{B})$, the $\log$ structure on the base must have characteristic $\mathbb{N}$. In fact, the issue is simply that $N_{S}$ is not a sheaf of saturated monoids. Saturating indeed gives $\bar{N}_{S}^{\text {sat }}=\mathbb{N}$ and corresponds to adding a generator $e^{\prime}$ such that $e=d e^{\prime}, e_{i}=l_{i} e^{\prime}$, with $d=\operatorname{l} \cdot \operatorname{c} \cdot \mathrm{m}\left(\alpha_{i}\right)$, $l_{i}=\frac{d}{\alpha_{i}}$. On the level of $\log$ structures (not just characteristics), consider the diagram

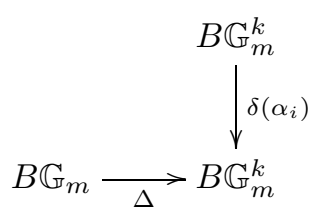

Here $B \mathbb{G}_{m}$ is viewed as the substack of $\mathcal{A}^{1}$ parametrizing pairs of line bundles with the zero section and $\delta\left(\alpha_{i}\right)$ is the morphism which, over a scheme $S$, sends a $k$-tuple of pairs of line bundles with their zero sections $\left(L_{i}, 0\right)$ to $\left(L_{i}^{\otimes \alpha_{i}}, 0\right)$ and $\Delta$ the diagonal morphism. We then have a morphism to the fiber product

$$
\mathcal{M}_{\Gamma_{1}}^{\log }(\mathcal{U} / \mathcal{B})^{\operatorname{sim}} \times_{D^{k}} \mathcal{M}_{\Gamma_{2}}^{\log }(\mathcal{U} / \mathcal{B})^{\sim} \rightarrow B \mathbb{G}_{m}^{k} \times{ }_{B \mathbb{G}_{m}^{k}} B \mathbb{G}_{m},
$$

which sends the data of the two maps to the collection

$$
\left(\left(N_{x_{i} / C_{1}} \otimes N_{x_{i} / C_{2}}, 0\right)_{i=1}^{k}, q_{\left.\right|_{D_{S}}}\left(N_{D_{S} / X_{1}} \otimes N_{D_{S} / X_{2}}\right), 0\right)
$$

and isomorphism (8). Then, saturating $N_{S}$ is equivalent to taking the fiber product

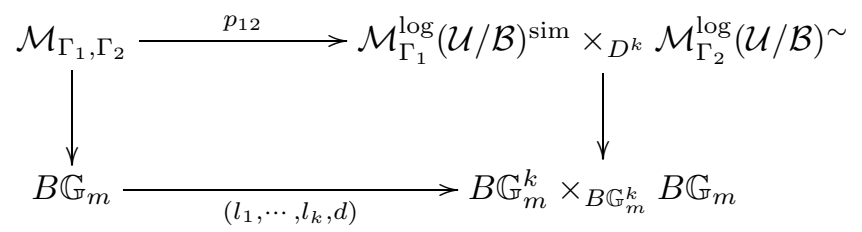

Lemma 5.2. The morphism $p_{12}: \mathcal{M}_{\Gamma_{1}, \Gamma_{2}} \rightarrow \mathcal{M}_{\Gamma_{1}}^{\log }(\mathcal{U} / \mathcal{B})^{\operatorname{sim}} \times_{D^{k}} \mathcal{M}_{\Gamma_{2}}^{\log }(\mathcal{U} / \mathcal{B})^{\sim}$ is finite étale and surjective of pure degree $\frac{\prod_{i=1}^{k} \alpha_{i}}{d}$. 
Proof. The lower arrow of the above cartesian square factors as

$$
B \mathbb{G}_{m} \stackrel{\left(l_{1}, \cdots, l_{k}, 1\right)}{\longrightarrow} B \mathbb{G}_{m}^{k} \underset{\delta\left(\alpha_{i}\right), B \mathbb{G}_{m}^{k}, d \Delta}{\times} B \mathbb{G}_{m} \stackrel{(1, \cdots, 1, d)}{\longrightarrow} B \mathbb{G}_{m}^{k} \underset{\delta\left(\alpha_{i}\right), B \mathbb{G}_{m}^{k}, \Delta}{\times} B \mathbb{G}_{m}
$$

where $d \Delta$ sends a pair of a line bundle with its zero section $(L, 0)$ to $k$ copies of $\left(L^{\otimes d}, 0\right)$. The second morphism is base change from the $d$-th power morphism $B \mathbb{G}_{m} \stackrel{d}{\rightarrow} B \mathbb{G}_{m}$, hence a gerbe banded by $\mu_{d}$. The first one is a $\prod_{i=1}^{k} \mu_{\alpha_{i}}$-torsor; indeed an $S$-point of the fiber product in the middle corresponds to line bundles $N_{i}, L, i=1, \ldots, k$ on $S$ with isomorphisms $\phi_{i}: N_{i}^{\alpha_{i}} \rightarrow L^{\otimes d}$. Then the pullback of $\left(l_{1}, \cdots, l_{k}, 1\right)$ via $S$ is equivalent to the fibered category associated to the functor on $S$-schemes that sends $T \rightarrow S$ to $k$-tuples of isomorphisms $\left.\left.N_{i}\right|_{T} \cong L\right|_{T} ^{\otimes l_{i}}$ whose $\alpha_{i}$-th power is $\left.\phi_{i}\right|_{T}$.

C. Gluing the forgetful morphisms. Recall that there exist forgetful morphisms $\tau^{\text {sim }}: \mathcal{M}_{\Gamma_{1}}^{\log }(\mathcal{U} / \mathcal{B})^{\operatorname{sim}} \rightarrow \mathcal{M B}^{\text {sim }}=\mathfrak{M}_{g_{1}, n_{1}+k}^{\log } \times X$ and $\tau^{\sim}: \mathcal{M}_{\Gamma_{2}}^{\log }(\mathcal{U} / \mathcal{B})^{\sim} \rightarrow$ $\mathcal{M B}^{\sim}=\mathfrak{M}_{g_{2}, n_{2}+k}^{\log } \times{ }_{\mathrm{LOG}}\left(\mathcal{B}^{\sim}\right)^{\text {etw }}$. There exists an analogous forgetful morphism from $\mathcal{M}_{\Gamma_{1}, \Gamma_{2}}$ to a stack $\mathcal{M B}^{\text {gl }}$, to be defined below, whose objects are obtained by appropriately gluing the objects of $\mathcal{M B}^{\text {sim }}$ and $\mathcal{M B}^{\sim}$. To be more precise, we define a stack $\mathcal{M B}^{\prime}$ via the fiber product

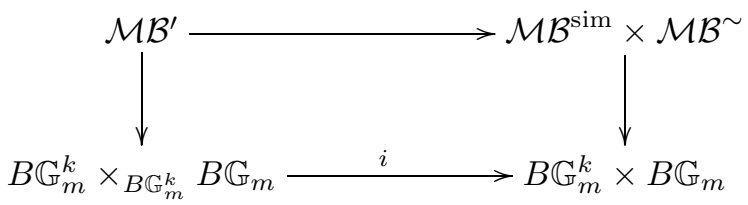

where $B \mathbb{G}_{m}^{k} \times{ }_{B \mathbb{G}_{m}^{k}} B \mathbb{G}_{m}$ is given by diagram (9) above. To define the right vertical arrow we glue the underlying scheme data of curves and targets of a pair of elements in $\mathcal{M B}^{\text {sim }}$ and $\mathcal{M B}^{\sim}$ over $S$ canonically along their $k$ sections and common distinguished divisor respectively. With notation as above, let the resulting glued pair have underlying scheme data $\left(C_{1},\left\{x_{i}\right\}_{i=0}^{k}, D_{S} \subset X_{1} \rightarrow S\right)$ and $\left(C_{2},\left\{x_{i}\right\}_{i=0}^{k}, D_{S} \subset X_{2} \rightarrow S\right)$; write $q_{\left.\right|_{D_{S}}}: D_{S} \rightarrow S$ for the glued family of divisors. Then we map the glued pair to

$$
\left\{\left(\left(N_{x_{i} / C_{1}} \otimes N_{x_{i} / C_{2}}\right)^{\alpha_{i}}, 0\right)_{i=1}^{k},\left(q_{\left.\right|_{D_{S}} *}\left(N_{D_{S} / X_{1}} \otimes N_{D_{S} / X_{2}}\right), 0\right)\right\} .
$$

Then $\mathcal{M B}^{\prime}$, over a scheme $S$, parametrizes pairs of elements of $\mathcal{M B}^{\text {sim }}$ and $\mathcal{M B}^{\sim}$, together with isomorphisms

$$
\psi_{i}:\left(\left(N_{x_{i} / C_{1}} \otimes N_{x_{i} / C_{2}}\right)^{\alpha_{i}}, 0\right) \rightarrow\left(q_{\left.\right|_{D_{S}}}\left(N_{D_{S} / X_{1}} \otimes N_{D_{S} / X_{2}}\right), 0\right) .
$$

Now the morphism $\mathcal{M}_{\Gamma_{1}}^{\log }(\mathcal{U} / \mathcal{B})^{\operatorname{sim}} \times_{D^{k}} \mathcal{M}_{\Gamma_{2}}^{\log }(\mathcal{U} / \mathcal{B})^{\sim} \rightarrow \mathcal{M B}^{\operatorname{sim}} \times \mathcal{M B}^{\sim}$ factors through $\mathcal{M B}^{\prime}$. Indeed, this follows from isomorphism (8), which was obtained as soon as the underlying maps were glued.

All in all, there is a stack $\mathcal{M B}^{\mathrm{gl}}$, which, over a scheme $S$, parametrizes

- a pair of elements of $\mathcal{M B}^{\mathrm{sim}}$ and $\mathcal{M B}^{\sim}$, with isomorphisms

$$
\psi_{i}:\left(\left(N_{x_{i} / C_{1}} \otimes N_{x_{i} / C_{2}}\right)^{\alpha_{i}}, 0\right) \rightarrow\left(q_{\left.\right|_{D_{S}} *}\left(N_{D_{S} / X_{1}} \otimes N_{D_{S} / X_{2}}\right), 0\right), i=1, \ldots, k ;
$$

- a line bundle $L$ on $S$ such that $L^{d} \cong q_{\left.\right|_{D_{S}}}\left(N_{D_{S} / X_{1}} \otimes N_{D_{S} / X_{2}}\right)$, and isomorphisms $\phi_{i}:\left(N_{x_{i} / C_{1}} \otimes N_{x_{i} / C_{2}}, 0\right) \cong\left(L^{l_{i}}, 0\right)$ such that $\phi_{i}^{\alpha_{i}}=\psi_{i}, i=1, \ldots, k$. 
Concretely, we have a diagram

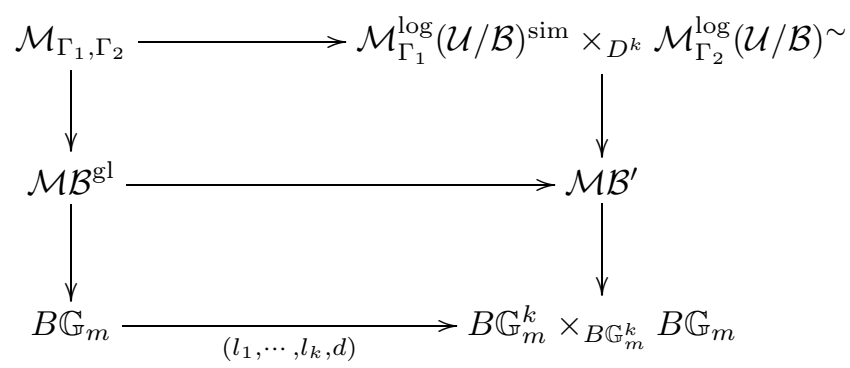

Here, the map $\left(l_{1}, \cdots, l_{k}, d\right)$ is precisely as in the previous section. The map $\mathcal{M B}^{\prime} \rightarrow B \mathbb{G}_{m}^{k} \times{ }_{B \mathbb{G}_{m}^{k}} B \mathbb{G}_{m}$ sends a pair of elements of $\mathcal{M B}^{\text {sim }}$ and $\mathcal{M B} \mathcal{B}^{\sim}$ over $S$, together with the isomorphisms $\psi_{i}$ defined above, to

$$
\left.\left\{\left(N_{x_{i} / C_{1}} \otimes N_{x_{i} / C_{2}}, 0\right),\left(q_{\left.\right|_{D_{S}}}\left(N_{D_{S} / X_{1}} \otimes N_{D_{S} / X_{2}}\right), 0\right), \psi_{i}\right)\right\}, i=1, \ldots, k .
$$

Consequently, the composed map $\mathcal{M}_{\Gamma_{1}}^{\log }(\mathcal{U} / \mathcal{B})^{\operatorname{sim}} \times_{D^{k}} \mathcal{M}_{\Gamma_{2}}^{\log }(\mathcal{U} / \mathcal{B})^{\sim} \rightarrow B \mathbb{G}_{m}^{k} \times{ }_{B \mathbb{G}_{m}^{k}}$ $B \mathbb{G}_{m}$ is the one considered in the previous section as well. From the fact that the bottom square in the diagram is cartesian, we conclude that $\mathcal{M B}^{\mathrm{gl}}$ is algebraic and the map $\mathcal{M B}^{\mathrm{gl}} \rightarrow \mathcal{M B}^{\prime}$ is étale of pure degree $\frac{\prod_{i=1}^{k} \alpha_{i}}{d}$. Furthermore, since the big square is cartesian, the top square is also cartesian.

Now let $\operatorname{Aut}\left(\Gamma_{1}, \Gamma_{2}\right)$ be the group of automorphisms of the partition $\vec{\alpha}$, that is, the number of bijections $\phi:[k] \rightarrow[k]$ with $\alpha_{\phi(i)}=\alpha_{i}$, and consider its natural action on $\mathcal{M}_{\Gamma_{1}, \Gamma_{2}}$. We then have a canonical identification

$$
F_{\Gamma_{1}, \Gamma_{2}}=\left[\frac{\mathcal{M}_{\Gamma_{1}, \Gamma_{2}}}{\operatorname{Aut}\left(\Gamma_{1}, \Gamma_{2}\right)}\right]
$$

of the fixed locus $F_{\Gamma_{1}, \Gamma_{2}}$ with the quotient stack of $\mathcal{M}_{\Gamma_{1}, \Gamma_{2}}$ by the (finite) group $\operatorname{Aut}\left(\Gamma_{1}, \Gamma_{2}\right)$ in the sense of Rom05. Finally, Aut $\left(\Gamma_{1}, \Gamma_{2}\right)$ also acts naturally on $\mathcal{M B}^{\mathrm{gl}}$, and, what is more, the image $\mathcal{M B}_{\Gamma_{1}, \Gamma_{2}}$ of the fixed locus $F_{\Gamma_{1}, \Gamma_{2}}$ in $\mathcal{M B}$ can be canonically identified with $\left[\frac{\mathcal{M B}^{\mathrm{gl}}}{\operatorname{Aut}\left(\Gamma_{1}, \Gamma_{2}\right)}\right]$. Let $q_{12}: \mathcal{M}_{\Gamma_{1}, \Gamma_{2}} \rightarrow F_{\Gamma_{1}, \Gamma_{2}}$ be the quotient map. We then have a cartesian diagram

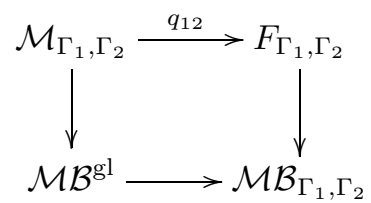

We thus have a description of the fixed loci in terms of known stacks. What is not a priori clear is the relation between the natural obstruction theory induced on $F_{\Gamma_{1}, \Gamma_{2}}$ by the obstruction theory of $\mathcal{M}_{\Gamma}^{\log }(\mathcal{U} / \mathcal{B})$ and those of the two factors $\mathcal{M}_{\Gamma_{1}}^{\log }(\mathcal{U} / \mathcal{B})^{\operatorname{sim}}, \mathcal{M}_{\Gamma_{2}}^{\log }(\mathcal{U} / \mathcal{B})^{\sim}$.

D. Comparison of obstruction theories. Let us denote by $\mathcal{T}_{f}^{1}$ and $\mathcal{T}_{f}^{2}$ the tangent and obstruction space of $\mathcal{M}_{\Gamma}^{\log }(\mathcal{U} / \mathcal{B})$ at a point $f$, as in Lemma 4.1, and by $\mathcal{T}_{i, f_{i}}^{1}, \mathcal{T}_{i, f_{i}}^{2}$ the tangent and obstruction spaces of the two component maps $f_{i}$. 
Lemma 5.3. At the point $f \in F_{\Gamma_{1}, \Gamma_{2}}$ we have the equality

$$
\mathcal{T}_{f}^{1}-\mathcal{T}_{f}^{2}=\mathcal{T}_{1, f_{1}}^{1}+\mathcal{T}_{2, f_{2}}^{1}-\mathcal{T}_{1, f_{1}}^{2}-\mathcal{T}_{2, f_{2}}^{2}-\left(T_{D}\right)^{k}+H^{0}\left(N_{\mathcal{D} / \mathcal{X}} \otimes N_{\mathcal{D} / \mathcal{Y}}\right)
$$

in K-theory.

Proof. Denote by $y_{1}, \cdots, y_{k}$ the nodes connecting $C_{1}$ with $C_{2}$, that is, the nodes over $D=D[0]$. Then $y_{1}, \cdots, y_{k}$ become marked points in $C_{1}, C_{2}$. We denote each of the two markings on $C_{1}$ and $C_{2}$ respectively over the node $y_{i}$ by the same symbol for ease of notation. Let $g=\pi \circ f: C \rightarrow W \rightarrow X$ be the composition of $f$ with the contraction $W \rightarrow X$, and let $g_{i}=\pi \circ f_{i}$. By Lemmas 4.1 and 4.2 combined we have that the fibers of $\mathcal{T}^{1}, \mathcal{T}^{2}$ fit into the six-term exact sequence

$$
0 \rightarrow \mathfrak{a u t}(\tau(f)) \rightarrow H^{0}\left(g^{*} T_{X}^{\log }\right) \rightarrow \mathcal{T}_{f}^{1} \rightarrow \operatorname{Def}(\tau(f)) \rightarrow H^{1}\left(g^{*} T_{X}^{\log }\right) \rightarrow \mathcal{T}_{f}^{2} \rightarrow 0 .
$$

We have two similar six-term exact sequences for $f_{i}$ with $C$ replaced by $C_{i}$ and $g$ by $g_{i}$ and $\tau$ replaced by $\tau^{\sim}$ for $f_{2}$. Write $\vec{x}$ for the vector of all marked points on $C$, as in Corollary 4.2 above, and $\left(S_{i}, y_{1}, \cdots, y_{k}\right)$ for the vector of marked points in $C_{i}$. Observe that

$$
\mathfrak{a u t}(C, \vec{x})=\mathfrak{a u t}\left(C_{1}, S_{1}, y_{1}, \cdots, y_{k}\right) \oplus \mathfrak{a u t}\left(C_{2}, y_{1}, \cdots, y_{k}, S_{2}\right)
$$

-vector fields that vanish on the nodes and marked points of $C$ are simply vector fields that vanish on the nodes and marked points of $C_{1}$ and $C_{2}$ and the nodes connecting the two. Furthermore,

$$
\mathfrak{a u t}_{\mathcal{B}}(W)=\mathfrak{a u t}_{\mathcal{B}} \sim(Y),
$$

and thus

$$
\mathfrak{a} \mathfrak{u t}(\tau(f))=\mathfrak{a u t}\left(\tau\left(f_{1}\right)\right) \oplus \mathfrak{a} \mathfrak{u}\left(\tau^{\sim}\left(f_{2}\right)\right) .
$$

Similarly, from the local-to-global sequence (6) we have an equality in $K$-theory

$$
\begin{aligned}
\operatorname{Ext}^{1}\left(\Omega_{\mathcal{C}}\left(\sum x_{i}\right), \mathcal{O}_{\mathcal{C}}\right)= & \operatorname{Ext}^{1}\left(\Omega_{\mathcal{C}_{1}}\left(\sum S_{1}+y_{1}+\cdots y_{k}\right), \mathcal{O}_{\mathcal{C}_{1}}\right) \\
& \bigoplus \operatorname{Ext}^{1}\left(\Omega_{\mathcal{C}_{2}}\left(\sum y_{i}+\sum S_{2}\right), \mathcal{O}_{\mathcal{C}_{2}}\right) \\
& \bigoplus_{i=1}^{k} T_{y_{i}} \mathcal{C}_{1} \otimes T_{y_{i}} \mathcal{C}_{2} .
\end{aligned}
$$

On the other hand, we have by Corollary 4.1 that $\operatorname{Def}(\tau(f))$ differs in $K$ theory from $\operatorname{Ext}^{1}\left(\Omega_{\mathcal{C}}\left(\sum x_{i}\right), \mathcal{O}_{\mathcal{C}}\right)$ by replacing $\bigoplus_{\text {nodes over D[i] }} T_{x} \mathcal{C}_{x}^{1} \otimes T_{x} \mathcal{C}_{x}^{2}$ with $H^{0}\left(N_{\mathcal{D}[i] / \mathcal{X}[i-1]} \otimes N_{\mathcal{D}[i] / \mathcal{X}[i]}\right)$. All nodes of $C$ persist as nodes over some target node in $C_{1}$ and $C_{2}$, except precisely the nodes over $D$, as $D$ is not a target node for either $f_{1}$ or $f_{2}$. Therefore,

$$
\operatorname{Def}(\tau(f))=\operatorname{Def}\left(\tau\left(f_{1}\right)\right) \oplus \operatorname{Def}\left(\tau^{\sim}\left(f_{2}\right)\right) \oplus H^{0}\left(N_{\mathcal{D} / \mathcal{X}} \otimes N_{\mathcal{D} / \mathcal{Y}}\right)
$$

It remains to analyze the relative deformations and obstructions of $f$ given by the cohomology groups $H^{i}\left(g^{*} T_{X}^{\log }\right)$. We have the normalization sequence

$$
0 \rightarrow \mathcal{O}_{C} \rightarrow \mathcal{O}_{C_{1}} \oplus \mathcal{O}_{C_{2}} \rightarrow \bigoplus_{i=1}^{k} \mathcal{O}_{y_{i}} \rightarrow 0
$$

By [Kat96, Example 10.2] and [Gro11, Example 3.31], the log tangent bundle $T_{X}(-\log D)$ fits into the short exact sequence

$$
0 \rightarrow T_{X}(-\log D) \rightarrow T_{X} \rightarrow N_{D / X} \rightarrow 0
$$


and thus coincides at a point of $D$ with the tangent space at that point in $D$. Therefore, twisting the normalization sequence by $g^{*} T_{X}^{\text {log }}$ and taking cohomology we get

$$
\begin{array}{r}
0 \rightarrow H^{0}\left(g^{*} T_{X}^{\log }\right) \rightarrow H^{0}\left(g_{1}^{*} T_{X}^{\log }\right) \oplus H^{0}\left(g_{2}^{*} T_{X}^{\log }\right) \rightarrow\left(T_{D}\right)^{k} \\
\rightarrow H^{1}\left(g^{*} T_{X}^{\log }\right) \rightarrow H^{1}\left(g_{1}^{*} T_{X}^{\log }\right) \oplus H^{1}\left(g_{2}^{*} T_{X}^{\log }\right) \rightarrow 0 .
\end{array}
$$

Therefore, the difference between $H^{0}\left(g^{*} T_{X}^{\log }\right)-H^{1}\left(g^{*} T_{X}^{\log }\right)$ and $\sum H^{0}\left(g_{i}^{*} T_{X}^{\log }\right)-$ $H^{1}\left(g_{i}^{*} T_{X}^{\log }\right)$ is precisely $\left(T_{D}\right)^{k}$. Putting everything together yields the lemma.

From the above lemma it follows that in $K$-theory the pullbacks of the (virtual) sheaves $\mathcal{T}^{1}-\mathcal{T}^{2}$ and $\sum \mathcal{T}_{i}^{1}-\mathcal{T}_{i}^{2}$ to $\mathcal{M}_{\Gamma_{1}, \Gamma_{2}}$ differ by two bundles: the first is the bundle with fiber $\left(T_{D}\right)^{k}$, which may be identified with the pullback of the tangent bundle $\left(T_{D}\right)^{k}$ under the evaluation map $\mathcal{M}_{\Gamma_{1}, \Gamma_{2}} \rightarrow \mathcal{M}_{\Gamma_{1}}^{\log }(\mathcal{U} / \mathcal{B})^{\operatorname{sim}} \times_{D^{k}}$ $\mathcal{M}_{\Gamma_{2}}^{\log }(\mathcal{U} / \mathcal{B})^{\sim} \rightarrow D^{k}$; the second one is the bundle with fiber $H^{0}\left(N_{\mathcal{D} / \mathcal{X}} \otimes N_{\mathcal{D} / \mathcal{Y}}\right)$. This is the line bundle $\mathcal{L}$ that parametrizes deformations of the node $D$; it may be identified with the pullback of $p_{1}^{*}\left(\mathcal{L}_{1}\right) \otimes p_{2}^{*}\left(\mathcal{L}_{2}\right)$ to $\mathcal{M}_{\Gamma_{1}, \Gamma_{2}}$, where $p_{1}$ and $p_{2}$ are the projections of $\mathcal{M}_{\Gamma_{1}}^{\log }(\mathcal{U} / \mathcal{B})^{\operatorname{sim}} \times_{D^{k}} \mathcal{M}_{\Gamma_{2}}^{\log }(\mathcal{U} / \mathcal{B})^{\sim}$ to the two factors, and $\mathcal{L}_{i}$ are the respective similar bundles. Note that $\mathcal{L}_{1}$ is a trivial bundle with nontrivial action, while $\mathcal{L}_{2}$ is a non-trivial bundle with trivial action. All the above sheaves on $\mathcal{M}_{\Gamma_{1}, \Gamma_{2}}$ descend to $F_{\Gamma_{1}, \Gamma_{2}}$; we will denote the sheaves descended from them by the same symbol. To keep consistent with existing literature, we write $e^{T}\left(\mathcal{L}_{1}\right)=\frac{w}{d}, e^{T}\left(\mathcal{L}_{2}\right)=-\frac{\psi}{d}$. We then obtain:

Corollary 5.3. If $F=F_{\Gamma_{1}, \Gamma_{2}}$ and $N_{\Gamma_{1}}=\left(\mathcal{T}_{1}^{1}-\mathcal{T}_{1}^{2}\right)^{\mathrm{m}}$, we have

$$
e^{T}\left(N_{F}^{\mathrm{vir}}\right)=e^{T}\left(N_{\Gamma_{1}}\right)\left(\frac{w-\psi}{d}\right) .
$$

Proof. Lemma 5.3 implies that $N_{F}^{\text {vir }}=\left(\mathcal{T}^{1}-\mathcal{T}^{2}\right)^{\mathrm{m}}$ differs from the sum of the $\left(\mathcal{T}_{i}^{1}-\mathcal{T}_{i}^{2}\right)^{\mathrm{m}}$ only by the bundle $\mathcal{L}$, since $T_{D}$ has trivial action. Furthermore, since the torus action on $\mathcal{M}_{\Gamma_{2}}^{\log }(\mathcal{U} / \mathcal{B}) \sim$ is trivial, the bundles $\mathcal{T}_{2}^{j}$ have no moving part.

The perfect obstruction theory of the fixed locus $F_{\Gamma_{1}, \Gamma_{2}}$ is by definition obtained by restriction from the torus fixed part of the perfect obstruction theory of $\mathcal{M}_{\Gamma}^{\log }(\mathcal{U} / \mathcal{B})$ relative to $\mathcal{M B}$ discussed in section 4. Recall that the image $\mathcal{M B}_{\Gamma_{1}, \Gamma_{2}}$ of $F_{\Gamma_{1}, \Gamma_{2}}$ in $\mathcal{M B}$ was identified with the étale quotient of the stack $\mathcal{M B}^{\mathrm{gl}}$ by the group $\operatorname{Aut}\left(\Gamma_{1}, \Gamma_{2}\right)$ (section 5.3.C). Now note that the relative cotangent complex of $\mathcal{M B}_{\Gamma_{1}, \Gamma_{2}}$ in $\mathcal{M B}$ has trivial torus-fixed part: the torus action on the deformation space of the $k$ nodes and the divisor obtained from gluing the objects in $\mathcal{M B}^{\text {sim }}$ and $\mathcal{M B}^{\sim}$ only has trivial fixed locus. Therefore $F_{\Gamma_{1}, \Gamma_{2}}$ has a perfect obstruction theory relative to its image, which yields the same virtual fundamental class $\left[F_{\Gamma_{1}, \Gamma_{2}}\right]^{\text {vir }}$. Consequently, in view of the cartesian diagram (10), we may pull back the above obstruction theory via the étale quotient map $q_{12}: \mathcal{M}_{\Gamma_{1}, \Gamma_{2}} \rightarrow F_{\Gamma_{1}, \Gamma_{2}}$ to obtain a perfect obstruction theory for $\mathcal{M}_{\Gamma_{1}, \Gamma_{2}}$ relative to $\mathcal{M B}^{\mathrm{gl}}$ and thus a virtual fundamental class $\left[\mathcal{M}_{\Gamma_{1}, \Gamma_{2}}\right]^{\text {vir }}$. Now, by $\operatorname{Cos} 06$, Theorem 5.0.1], we have

$$
q_{12 *}\left[\mathcal{M}_{\Gamma_{1}, \Gamma_{2}}\right]^{\mathrm{vir}}=\left|\operatorname{Aut}\left(\Gamma_{1}, \Gamma_{2}\right)\right|\left[F_{\Gamma_{1}, \Gamma_{2}}\right]^{\mathrm{vir}} .
$$


We have the following cartesian diagram

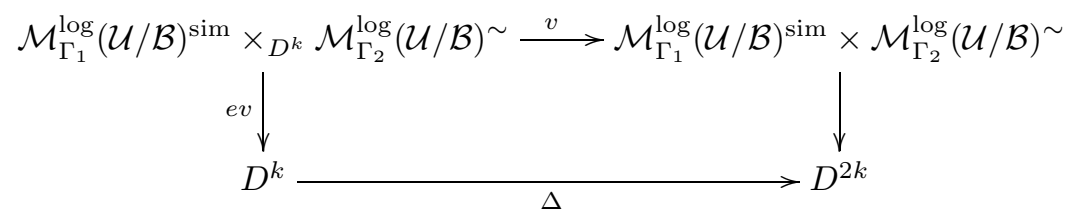

Now let $p_{12}: \mathcal{M}_{\Gamma_{1}, \Gamma_{2}} \rightarrow \mathcal{M}_{\Gamma_{1}}^{\log }(\mathcal{U} / \mathcal{B})^{\operatorname{sim}} \times_{D^{k}} \mathcal{M}_{\Gamma_{2}}^{\text {log }}(\mathcal{U} / \mathcal{B})^{\sim}$ be the morphism defined right before Lemma 5.2. Putting everything together, we obtain

Theorem 5.1 (Log Virtual Localization).

$$
\begin{aligned}
{\left[\mathcal{M}_{\Gamma}^{\log }(\mathcal{U} / \mathcal{B})\right]^{\text {vir }}=} & {\left[\mathcal{M}_{\Gamma}^{\log }(\mathcal{U} / \mathcal{B})^{\operatorname{sim}}\right]^{\text {vir }} } \\
& +\sum_{\Gamma_{1}, \Gamma_{2}} \frac{q_{12 *} p_{12}^{*} \Delta^{!}\left(\left[\mathcal{M}_{\Gamma_{1}}^{\log }(\mathcal{U} / \mathcal{B})^{\operatorname{sim}}\right]^{\text {vir }} \times\left[\mathcal{M}_{\Gamma_{2}}^{\log }(\mathcal{U} / \mathcal{B})^{\sim}\right]^{\text {vir }}\right)}{\left|\operatorname{Aut}\left(\Gamma_{1}, \Gamma_{2}\right)\right|\left(\frac{w-\psi}{d}\right) e^{T}\left(N_{\Gamma_{1}}\right)}
\end{aligned}
$$

In the statement of the theorem the symbols $q_{12 *}, p_{12}^{*}$, and $\Delta^{!}$denote the proper pushforward, flat, and Gysin pullback operations, respectively, on the Chow groups of the corresponding Deligne-Mumford stacks (see [Vis89]).

Proof. It is enough to show that

$$
\left[\mathcal{M}_{\Gamma_{1}, \Gamma_{2}}\right]^{\text {vir }}=p_{12}^{*} \Delta^{!}\left(\left[\mathcal{M}_{\Gamma_{1}}^{\log }(\mathcal{U} / \mathcal{B})^{\operatorname{sim}}\right]^{\text {vir }} \times\left[\mathcal{M}_{\Gamma_{2}}^{\log }(\mathcal{U} / \mathcal{B})^{\sim}\right]^{\text {vir }}\right) .
$$

Then the theorem will follow immediately from equality (11) and Lemma 5.3 .

First, we claim that $\mathcal{M}_{\Gamma_{1}, \Gamma_{2}}$ has a perfect obstruction theory relative to $\mathcal{M B}^{\text {sim }} \times$ $\mathcal{M B}^{\sim}$, which yields the same virtual fundamental class $\left[\mathcal{M}_{\Gamma_{1}, \Gamma_{2}}\right]^{\text {vir }}$ as its perfect obstruction theory relative to $\mathcal{M B}^{\text {gl }}$ discussed above. The reason is that the relative cotangent complex of $\mathcal{M B}^{\mathrm{gl}}$ over $\mathcal{M B}^{\mathrm{sim}} \times \mathcal{M B}^{\sim}$ has trivial torusfixed part. Indeed, since $\mathcal{M B}^{\text {gl }}$ is étale over $\mathcal{M B}^{\prime}$, it suffices to consider the action of $\mathbb{C}^{*}$ on the cotangent bundle of the fibers of $\mathcal{M B}^{\prime} \rightarrow \mathcal{M B}^{\text {sim }} \times \mathcal{M B} \mathcal{B}^{\sim}$. In the notation of section 5.3.C, consider an element of $\mathcal{M B}^{\prime}(\mathbb{C})$ with underlying scheme data $\left(C_{1},\left\{x_{i}\right\}, D \subset X\right)$ and $\left(C_{1},\left\{x_{i}\right\}, D \subset Y\right)$ and isomorphisms $\psi_{i}:\left(N_{x_{i} / C_{1}} \otimes N_{x_{i} / C_{2}}\right)^{\alpha_{i}} \rightarrow q_{D *}\left(N_{D / X} \otimes N_{D / Y}\right)=\mathbb{C}, i=1, \ldots, k$. Then an element $c \in \mathbb{C}^{*}$ acts on $\psi_{i}$ by multiplying them by $c$, so its $\mathbb{C}^{*}$-action on the cotangent bundle of $\operatorname{Isom}\left(\left(N_{x_{i} / C_{1}} \otimes N_{x_{i} / C_{2}}\right)^{\alpha_{i}}, q_{D_{*}}\left(N_{D / X} \otimes N_{D / Y}\right)\right)$ is non-trivial.

We have a commutative diagram

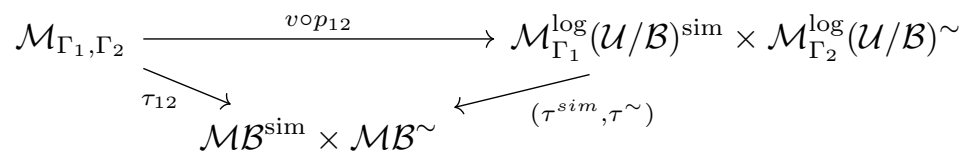

where $\tau_{12}$ is the composite morphism $\mathcal{M}_{\Gamma_{1}, \Gamma_{2}} \rightarrow \mathcal{M B}^{g l} \rightarrow \mathcal{M B}^{\sim} \times \mathcal{M B}^{\text {sim }}, \tau^{\text {sim }}, \tau^{\sim}$ the forgetful morphisms, and $v$ the morphism defined in diagram (12). By the previous paragraph and the proof of Lemma 5.3, the deformation and obstruction spaces of the map $f \in \mathcal{M}_{\Gamma_{1}, \Gamma_{2}}$ and its component maps $\left(f_{1}, f_{2}\right) \in \mathcal{M}_{\Gamma_{1}}^{\log }(\mathcal{U} / \mathcal{B})^{\operatorname{sim}} \times$ $\mathcal{M}_{\Gamma_{2}}^{\log }(\mathcal{U} / \mathcal{B})^{\sim}$, over the same point in $\mathcal{M B}^{\text {sim }} \times \mathcal{M B}^{\sim}$, differ by the term $T_{D}^{k}$. In the formalism of Manolache Man12, Definition 4.5], this is equivalent to the statement that the perfect obstruction theories of $\tau_{12}$ and $\left(\tau^{\mathrm{sim}}, \tau^{\sim}\right)$ together with the trivial 
obstruction theory of $v \circ p_{12}$, given by its relative cotangent complex $\mathcal{L}_{v \circ p_{12}}=$ $p_{12}^{*} e v^{*} T_{D}^{k}$, form a compatible triple. Clearly $v \circ p_{12}$ is of Deligne-Mumford type, and the same argument as in [AMW12, Lemma 4.2.1] shows that $\left(\tau^{\operatorname{sim}}, \tau^{\sim}\right)$ is also of Deligne-Mumford type. Then, using [Man12, Lemma 4.9], we deduce that $\left[\mathcal{M}_{\Gamma_{1}, \Gamma_{2}}\right]^{\text {vir }}$ is the virtual pullback of $\left[\mathcal{M}_{\Gamma_{1}}^{\log }(\mathcal{U} / \mathcal{B})^{\operatorname{sim}}\right]^{\text {vir }} \times\left[\mathcal{M}_{\Gamma_{2}}^{\log }(\mathcal{U} / \mathcal{B})^{\sim}\right]^{\text {vir }}([$ Man12, Definition 3.7]]) with respect to $\mathcal{L}_{v \circ p_{12}}$, which, in our case, is simply its Gysin pullback via $\Delta$ followed by the flat pullback $p_{12}^{*}$ (see Remarks 3.9 and 3.10 in Man12]).

In section 2, we discussed the finite morphism $\pi: \mathcal{M}_{\Gamma}^{\log }(\mathcal{U} / \mathcal{B}) \rightarrow \mathcal{M}_{\Gamma}(X, D)$ from the moduli space of log stable maps to the moduli space of relative stable maps. In the paper AMW12 it is shown that the pushforward of the virtual fundamental class of $\mathcal{M}_{\Gamma}^{\log }(\mathcal{U} / \mathcal{B})$ under $\pi$ coincides with the virtual fundamental class of Jun Li's space. We may modify these results to include the maps $\pi^{\text {sim }}$ : $\mathcal{M}_{\Gamma_{1}}^{\log }(\mathcal{U} / \mathcal{B})^{\operatorname{sim}} \rightarrow \mathcal{M}_{\Gamma_{1}}^{\operatorname{sim}}(X, D)=\mathcal{M}_{\Gamma_{1}}^{\text {sim }}$ and $\pi^{\sim}: \mathcal{M}_{\Gamma_{2}}^{\log }(\mathcal{U} / \mathcal{B})^{\sim} \rightarrow \mathcal{M}_{\Gamma_{2}}^{\widetilde{N}_{2}}$, with the appropriate modifications of the spaces in the setting of relative stable maps as targets. Then, applying $\pi_{*}$ to both sides of the equation in Theorem 5.1 yields the relative virtual localization theorem of Graber-Vakil.

Corollary 5.4. The log virtual localization formula becomes the relative virtual localization formula under the functor $\pi_{*}$.

Proof. First, note that the fixed locus $F_{\Gamma_{1}, \Gamma_{2}}^{\mathrm{Li}}$ of Li's space corresponding to the splitting data $\left(\Gamma_{1}, \Gamma_{2}\right)$ is identified as the quotient stack of $\mathcal{M}_{\Gamma_{1}}^{\text {sim }} \times_{D^{k}} \mathcal{M}_{\Gamma_{1}}$ by $\operatorname{Aut}\left(\Gamma_{1}, \Gamma_{2}\right)$ GV05, page 13]; let $q_{12}^{\mathrm{Li}}$ be the quotient map. Then we have a commutative diagram

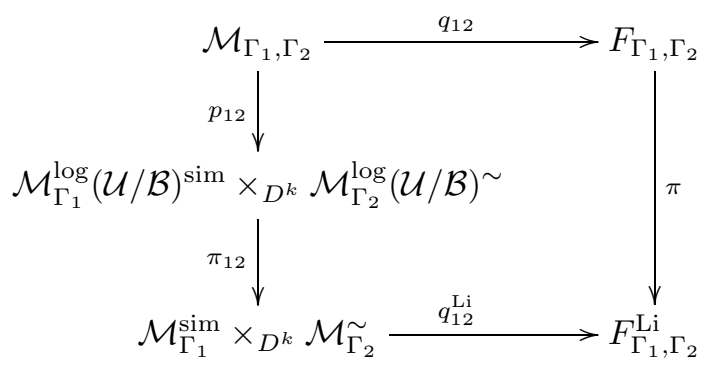

where $\pi_{12}$ is the finite map induced by $\pi^{\operatorname{sim}}$ and $\pi^{\sim}$. Therefore, the effect of $\pi_{*}$ on the numerators appearing on the right-hand side of the formula is the same as the effect of $q_{12 *}^{\mathrm{Li}} \pi_{12 *} p_{12 *}$ on $p_{12}^{*} \Delta^{!}\left(\left[\mathcal{M}_{\Gamma_{1}}^{\log }(\mathcal{U} / \mathcal{B})^{\operatorname{sim}}\right]^{\text {vir }} \times\left[\mathcal{M}_{\Gamma_{2}}^{\log }(\mathcal{U} / \mathcal{B})^{\sim}\right]^{\text {vir }}\right)$. By the projection formula and Lemma [5.2] we have

$$
\begin{aligned}
& p_{12 *} p_{12}^{*} \Delta^{!}\left(\left[\mathcal{M}_{\Gamma_{1}}^{\log }(\mathcal{U} / \mathcal{B})^{\operatorname{sim}}\right]^{\text {vir }} \times\left[\mathcal{M}_{\Gamma_{2}}^{\log }(\mathcal{U} / \mathcal{B})^{\sim}\right]^{\text {vir }}\right) \\
& =\frac{\prod \alpha_{i}}{d} \Delta^{!}\left(\left[\mathcal{M}_{\Gamma_{1}}^{\log }(\mathcal{U} / \mathcal{B})^{\operatorname{sim}}\right]^{\operatorname{vir}} \times\left[\mathcal{M}_{\Gamma_{2}}^{\log }(\mathcal{U} / \mathcal{B})^{\sim}\right]^{\text {vir }}\right)
\end{aligned}
$$


Now, we have the cartesian diagram

$$
\begin{aligned}
\mathcal{M}_{\Gamma_{1}}^{\log }(\mathcal{U} / \mathcal{B})^{\operatorname{sim}} \times_{D^{k}} \mathcal{M}_{\Gamma_{2}}^{\log }(\mathcal{U} / \mathcal{B})^{\sim} & \longrightarrow \mathcal{M}_{\Gamma_{1}}^{\log }(\mathcal{U} / \mathcal{B})^{\operatorname{sim}} \times \mathcal{M}_{\Gamma_{2}}^{\log }(\mathcal{U} / \mathcal{B})^{\sim} \\
\pi_{12} \downarrow & \downarrow{\left(\pi^{\operatorname{sim}}, \pi^{\sim}\right)}^{\sim} \\
\mathcal{M}_{\Gamma_{1}}^{\operatorname{sim}} \times{ }_{D^{k}} \mathcal{M}_{\Gamma_{2}}^{\sim} & \longrightarrow \mathcal{M}_{\Gamma_{1}}^{\operatorname{sim}} \times \mathcal{M}_{\Gamma_{2}}^{\sim}
\end{aligned}
$$

over $\Delta: D^{k} \rightarrow D^{2 k}$. Therefore, by [Vis89, Theorem 3.12] and the results of AMW12, we get

$$
\pi_{12 *} \Delta^{!}\left(\left[\mathcal{M}_{\Gamma_{1}}^{\log }(\mathcal{U} / \mathcal{B})^{\operatorname{sim}}\right]^{\text {vir }} \times\left[\mathcal{M}_{\Gamma_{2}}^{\log }(\mathcal{U} / \mathcal{B})^{\sim}\right]^{\text {vir }}\right)=\Delta^{!}\left[\mathcal{M}_{\Gamma_{1}}^{\operatorname{sim}} \times \mathcal{M}_{\Gamma_{2}}^{\sim}\right]^{\text {vir }}
$$

which, in turn, after applying $q_{12}{ }_{*}^{\mathrm{Li}}$ gives $\left|\operatorname{Aut}\left(\Gamma_{1}, \Gamma_{2}\right)\right|\left[F_{\Gamma_{1}, \Gamma_{2}}^{\mathrm{Li}}\right]^{\mathrm{vir}}$ by GV05, Lemma $3.2]$.

So what remains is to analyze the Euler classes appearing in the denominators of the formula. The term $e^{T}\left(N_{\Gamma}\right)$ does not change under $\pi$, as it is the Euler class of the virtual normal bundle of a fixed locus inside the simple locus, and $\pi$ is an isomorphism over the simple locus. On the other hand, let $L$ be the line bundle in $\mathcal{M}_{\Gamma}(X, D)$ parametrizing deformations of the node; its fiber at a point is $H^{0}\left(C, N_{D / X} \otimes N_{D / Y}\right)$. We denote the Euler class of $L$ by $w-\psi$, as in GV05. Note that over a fixed locus $F_{\Gamma_{1}, \Gamma_{2}}$, the pullback $\pi^{*}(L) \cong \mathcal{L}^{d}$, where $\mathcal{L}$ is the line bundle of $\mathcal{M}_{\Gamma}^{\log }(\mathcal{U} / \mathcal{B})$ with fiber $H^{0}\left(N_{\mathcal{D} / \mathcal{X}} \otimes N_{\mathcal{D} / \mathcal{Y}}\right)$ parametrizing deformations of the node $\mathcal{D}$, which is the $d$-th root of $D$. We thus have $e^{T}\left(\pi^{*} L\right)=d e^{T}(\mathcal{L})=d\left(\frac{w-\psi}{d}\right)$, which justifies the choice of notation for $e^{T}(\mathcal{L})$.

Summing over all $\Gamma_{1}, \Gamma_{2}$ gives precisely the relative virtual localization formula of GV05.

\section{ACKNOWLEDGments}

We are grateful to our advisor, Dan Abramovich, for suggesting this problem to us and for numerous valuable discussions. We also thank Angelo Vistoli for providing the idea of the proof of Lemma 3.1. Finally, we would like to thank the anonymous referee for useful comments.

\section{REFERENCES}

[AC11] Dan Abramovich and Qile Chen, Stable logarithmic maps to Deligne-Faltings pairs II, Asian J. Math. 18 (2014), no. 3, 465-488, DOI 10.4310/AJM.2014.v18.n3.a5. MR 3257836

[AMW12] Dan Abramovich, Steffen Marcus, and Jonathan Wise, Comparison theorems for Gromov-Witten invariants of smooth pairs and of degenerations (English, with English and French summaries), Ann. Inst. Fourier (Grenoble) 64 (2014), no. 4, 1611-1667. MR 3329675

[AV02] Dan Abramovich and Angelo Vistoli, Compactifying the space of stable maps, J. Amer. Math. Soc. 15 (2002), no. 1, 27-75, DOI 10.1090/S0894-0347-01-00380-0. MR1862797

[BV12] Niels Borne and Angelo Vistoli, Parabolic sheaves on logarithmic schemes, Adv. Math. 231 (2012), no. 3-4, 1327-1363, DOI 10.1016/j.aim.2012.06.015. MR2964607

[Che14] Qile Chen, Stable logarithmic maps to Deligne-Faltings pairs I, Ann. of Math. (2) 180 (2014), no. 2, 455-521, DOI 10.4007/annals.2014.180.2.2. MR.3224717

[CKL17] Huai-Liang Chang, Young-Hoon Kiem, and Jun Li, Torus localization and wall crossing for cosection localized virtual cycles, Adv. Math. 308 (2017), 964-986, DOI 10.1016/j.aim.2016.12.019. MR3600080 
[Cos06] Kevin Costello, Higher genus Gromov-Witten invariants as genus zero invariants of symmetric products, Ann. of Math. (2) 164 (2006), no. 2, 561-601, DOI 10.4007/annals.2006.164.561. MR.2247968

[Gil11] W. D. Gillam, Logarithmic stacks and minimality, Internat. J. Math. 23 (2012), no. 7, 1250069, 38, DOI 10.1142/S0129167X12500693. MR.2945649

[GP99] T. Graber and R. Pandharipande, Localization of virtual classes, Invent. Math. 135 (1999), no. 2, 487-518, DOI 10.1007/s002220050293. MR.1666787

[Gro11] Mark Gross, Tropical geometry and mirror symmetry, CBMS Regional Conference Series in Mathematics, vol. 114, Published for the Conference Board of the Mathematical Sciences, Washington, DC; by the American Mathematical Society, Providence, RI, 2011. MR 2722115

[GS11] Mark Gross and Bernd Siebert, Logarithmic Gromov-Witten invariants, J. Amer. Math. Soc. 26 (2013), no. 2, 451-510, DOI 10.1090/S0894-0347-2012-00757-7. MR.3011419

[GV05] Tom Graber and Ravi Vakil, Relative virtual localization and vanishing of tautological classes on moduli spaces of curves, Duke Math. J. 130 (2005), no. 1, 1-37, DOI 10.1215/S0012-7094-05-13011-3. MR2176546

[IP03] Eleny-Nicoleta Ionel and Thomas H. Parker, Relative Gromov-Witten invariants, Ann. of Math. (2) 157 (2003), no. 1, 45-96, DOI 10.4007/annals.2003.157.45. MR.1954264

[IP04] Eleny-Nicoleta Ionel and Thomas H. Parker, The symplectic sum formula for GromovWitten invariants, Ann. of Math. (2) 159 (2004), no. 3, 935-1025, DOI 10.4007/annals.2004.159.935. MR2113018

[Kat] K. Kato, Logarithmic degeneration and Dieudonne theory, preprint.

[Kat96] Fumiharu Kato, Log smooth deformation theory, Tohoku Math. J. (2) 48 (1996), no. 3, 317-354, DOI 10.2748/tmj/1178225336. MR:1404507

[Kat00] Fumiharu Kato, Log smooth deformation and moduli of log smooth curves, Internat. J. Math. 11 (2000), no. 2, 215-232, DOI 10.1142/S0129167X0000012X. MR.1754621

[Kim10] Bumsig Kim, Logarithmic stable maps, New developments in algebraic geometry, integrable systems and mirror symmetry (RIMS, Kyoto, 2008), Adv. Stud. Pure Math., vol. 59, Math. Soc. Japan, Tokyo, 2010, pp. 167-200. MR2683209

[Kon95] Maxim Kontsevich, Enumeration of rational curves via torus actions, The moduli space of curves (Texel Island, 1994), Progr. Math., vol. 129, Birkhäuser Boston, Boston, MA, 1995, pp. 335-368, DOI 10.1007/978-1-4612-4264-2_12. MR1363062

[Li01] Jun Li, Stable morphisms to singular schemes and relative stable morphisms, J. Differential Geom. 57 (2001), no. 3, 509-578. MR1882667

[Li02] Jun Li, A degeneration formula of $G W$-invariants, J. Differential Geom. 60 (2002), no. 2, 199-293. MR1938113

[LR01] An-Min Li and Yongbin Ruan, Symplectic surgery and Gromov-Witten invariants of Calabi-Yau 3-folds, Invent. Math. 145 (2001), no. 1, 151-218, DOI 10.1007/s002220100146. MR.1839289

[Man12] Cristina Manolache, Virtual pull-backs, J. Algebraic Geom. 21 (2012), no. 2, 201-245, DOI 10.1090/S1056-3911-2011-00606-1. MR2877433

[MFK94] D. Mumford, J. Fogarty, and F. Kirwan, Geometric invariant theory, 3rd ed., Ergebnisse der Mathematik und ihrer Grenzgebiete (2) [Results in Mathematics and Related Areas (2)], vol. 34, Springer-Verlag, Berlin, 1994. MR1304906

[Niz06] Wiesława Niziol, Toric singularities: log-blow-ups and global resolutions, J. Algebraic Geom. 15 (2006), no. 1, 1-29, DOI 10.1090/S1056-3911-05-00409-1. MR2177194

[Ols03] Martin C. Olsson, Universal log structures on semi-stable varieties, Tohoku Math. J. (2) $5 \mathbf{5}$ (2003), no. 3, 397-438. MR1993863

[Ols07] Martin C. Olsson, (Log) twisted curves, Compos. Math. 143 (2007), no. 2, 476-494, DOI 10.1112/S0010437X06002442. MR2309994

[Rom05] Matthieu Romagny, Group actions on stacks and applications, Michigan Math. J. 53 (2005), no. 1, 209-236, DOI 10.1307/mmj/1114021093. MR2125542

[Tot04] Burt Totaro, The resolution property for schemes and stacks, J. Reine Angew. Math. 577 (2004), 1-22, DOI 10.1515/crll.2004.2004.577.1. MR2108211

[Vis89] Angelo Vistoli, Intersection theory on algebraic stacks and on their moduli spaces, Invent. Math. 97 (1989), no. 3, 613-670, DOI 10.1007/BF01388892. MR1005008 
Department of Mathematics, University of Colorado Boulder, Campus Box 395, Boulder, Colorado 80309-0395; and Mathematical Research Center, Scuola Normale Superiore di Pisa 56126 Pisa, ItAly

Email address: samouil.molcho@sns.it

Department of Mathematics, Brown University, Box 1917, 151 Thayer Street, Providence, Rhode Island 02912; And Kavli IPMU (WPI), UTIAS, The University of Tokyo, KAshiwa, ChiBA 277-8583, JAPAN

Current address: Max Planck Institute for Mathematics, Vivatsgasse 7, 53111 Bonn, Germany

Email address: evangelos.routis@ipmu.jp, routis@mpim-bonn.mpg.de 